\title{
Polarized orbifolds associated to quantized Hamiltonian torus actions
}

\author{
Roberto Paoletti *
}

\begin{abstract}
Suppose given an holomorphic and Hamiltonian action of a compact torus $T$ on a polarized Hodge manifold $M$. Assume that the action lifts to the quantizing line bundle, so that there is an induced unitary representation of $T$ on the associated Hardy space. If in addition the moment map is nowhere zero, for each weight $\boldsymbol{\nu}$ the $\boldsymbol{\nu}$-th isotypical component in the Hardy space of the polarization is finitedimensional. Assuming that the moment map is transverse to the ray through $\boldsymbol{\nu}$, we give a gometric interpretation of the isotypical components associated to the weights $k \boldsymbol{\nu}, k \rightarrow+\infty$, in terms of certain polarized orbifolds associated to the Hamiltonian action and the weight. These orbifolds are generally not reductions of $M$ in the usual sense, but arise rather as quotients of certain loci in the unit circle bundle of the polarization; this construction generalizes the one of weighted projective spaces as quotients of the unit sphere, viewed as the domain of the Hopf map.
\end{abstract}

\section{Introduction}

Let $M$ be a $d$-dimensional connected complex projective manifold, with complex structure $J$. Let $(A, h)$ be a positive holomorphic line bundle on $(M, J)$; the curvature of the unique covariant derivative on $A$ compatible with both the Hermitian metric $h$ and the complex structures has the form $\Theta=-2 \pi \imath \omega$, where $\omega$ is a Kähler form on $(M, J)$. Let $\mathrm{d} V_{M}:=\omega^{\wedge d} / d$ ! be the associated volume form on $M$.

Let $A^{\vee}$ be the dual line bundle of $A$, endowed with the dual Hermitian metric $h^{\vee}$. As is well-known, positivity of $(A, h)$ is equivalent to the unit

\footnotetext{
*Address: Dipartimento di Matematica e Applicazioni, Università degli Studi di Milano-Bicocca, Via R. Cozzi 55, 20125 Milano, Italy; e-mail: roberto.paoletti@unimib.it
} 
disc bundle $D \subset A^{\vee}$ being a strictly pseudoconvex domain $[\mathrm{Gr}$. We shall denote by $X:=\partial D \subset A^{\vee}$ the unit circle bundle of $h^{\vee}$, and by $\alpha \in \Omega^{1}(X)$ the (normalized) connection 1-form on $X$. Thus, $X$ is a principal $S^{1}$-bundle on $M$, with the structure $S^{1}$-action $\rho^{X}: S^{1} \times X \rightarrow X$ given by clockwise fiber rotation. If $\pi: X \rightarrow M$ is the bundle projection, and $-\partial_{\theta} \in \mathfrak{X}(X)$ is the generator of $\rho^{X}$, then

$$
\mathrm{d} \alpha=2 \pi^{*}(\omega), \quad \alpha\left(\partial_{\theta}\right)=1
$$

Let $\mathrm{d} V_{X}:=(\alpha / 2 \pi) \wedge \pi^{*}\left(\mathrm{~d} V_{M}\right)$ be the associated volume form on $X$

Then $\alpha$ is a contact form on $X$, and $X$ is a CR manifold, with CR structure supported by the horizontal tangent bundle

$$
\operatorname{Hor}(X):=\operatorname{ker}(\alpha) \subset T X \text {. }
$$

Let $H(X) \subseteq L^{2}(X)$ denote the Hardy space of $X$. Since $\rho^{X}$ preserves $\alpha$ and the CR structure, it induces a unitary representation $\hat{\rho}^{X}$ of $S^{1}$ on $H(X)$, given by

$$
\hat{\rho}_{e^{\imath \vartheta}}^{X}(s)(x):=s\left(\rho_{e^{-\imath \vartheta}}^{X}(x)\right)=s\left(e^{\imath \vartheta} x\right) \quad\left(x \in X, e^{\imath \vartheta} \in S^{1}, s \in H(X)\right) .
$$

The induced isotypical decomposition is the Hilbert space direct sum

$$
H(X)=\bigoplus_{k=0}^{+\infty} H(X)_{k}
$$

where

$$
H(X)_{k}:=\left\{s \in H(X): s\left(e^{\imath \theta} x\right)=e^{\imath k \theta} s(x) \quad \forall x \in X, e^{\imath \theta} \in S^{1}\right\} .
$$

It is well-known that there are natural unitary isomorphisms $H(X)_{k} \cong$ $H^{0}\left(M, A^{\otimes k}\right)$, the latter being the space of global holomorphic sections of $A^{\otimes k}$.

Furthermore, let $T \cong\left(S^{1}\right)^{r}$ be an $r$-dimensional compact torus, with Lie algebra and coalgebra $\mathfrak{t}$ and $\mathfrak{t}^{\vee}$, respectively. We shall equivariantly identify $\mathfrak{t} \cong \mathfrak{t}^{\vee} \cong \imath \mathbb{R}^{r}$. Suppose given an Hamiltonian and holomorphic action $\mu^{M}: T \times M \rightarrow M$ of $T$ on the Kähler manifold $(M, J, 2 \omega)$. Let $\Phi: M \rightarrow \mathfrak{t}^{\vee} \cong \imath \mathbb{R}^{r}$ be the moment map.

It is standard that $\mu^{M}$ and $\Phi$ generate an infinitesimal contact and CR action of $\mathfrak{t}$ on $X$, so defined [Ko]. If $\xi \in \mathfrak{t}$, let $\xi_{M} \in \mathfrak{X}(M)$ be the Hamiltonian vector field induced by $\xi$ on $M$, and define a vector field $\xi_{X} \in \mathfrak{X}(X)$ by setting

$$
\xi_{X}:=\xi_{M}^{\sharp}-\langle\Phi \circ \pi, \xi\rangle \partial_{\theta} \in \mathfrak{X}(X) ;
$$


here $V^{\sharp} \in \mathfrak{X}(X)$ denotes the horizontal lift to $X$ of a vector field $V \in \mathfrak{X}(M)$, with respect to $\alpha$. The $\xi_{X}$ 's are commuting contact vector fields on $X$, whose flow preserves the CR structure, and the map $\xi \mapsto \xi_{X}$ is a morphism of Lie algebras $\mathfrak{t} \rightarrow \mathfrak{X}(X)$.

Let us make the stronger hypothesis that $\mu^{M}$ lifts to an actual contact and CR action of $T$ on $X, \mu^{X}: T \times X \rightarrow X$, and that $\mathrm{d} \mu^{X}(\xi)=\xi_{X}$ for any $\xi \in \mathfrak{t}$. Then $\mu^{X}$ determines a unitary representation $\hat{\mu}^{X}$ of $T$ on $H(X)$, given by

$$
\hat{\mu}_{\mathbf{t}}^{X}(s)(x):=s\left(\mu_{\mathbf{t}^{-1}}^{X}(x)\right) \quad(x \in X, \mathbf{t} \in T, s \in H(X)) .
$$

By the Peter-Weyl Theorem [St], $\hat{\mu}^{X}$ induces a unitary and equivariant splitting of $H(X)$ into isotypical components.

Let us regard any $\boldsymbol{\nu} \in \mathbb{Z}^{r}$ as an integral weight on $T$, associated to the character

$$
\chi_{\nu}(\mathbf{t}):=\mathbf{t}^{\nu},
$$

where for $\mathbf{t}=\left(t_{1}, \ldots, t_{r}\right) \in T$ we set $\mathbf{t}^{\nu}:=\prod_{j=1}^{r} t_{j}^{\nu_{j}}$. For any $\boldsymbol{\nu} \in \mathbb{Z}^{r}$, let us consider the $\boldsymbol{\nu}$-th isotypical component

$$
H(X)_{\nu}^{\hat{\mu}}:=\left\{s \in H(X): \hat{\mu}_{\mathbf{t}}(s)=\chi_{\boldsymbol{\nu}}(\mathbf{t}) \cdot s \quad \forall \mathbf{t} \in T\right\} .
$$

Then we have an equivariant Hilbert space direct sum

$$
H(X)=\bigoplus_{\nu \in \mathbb{Z}^{r}} H(X)_{\nu}^{\hat{\mu}}
$$

In the special case where $T=S^{1}, \mu^{M}$ is trivial, and $\Phi=\imath, \imath \in \mathfrak{t}$ is mapped to $-\partial_{\theta}$, and so $\mu^{X}=\rho^{X}$; hence (6) reduces to (3) , that is, $H(X)_{k}=H(X)_{k}^{\hat{\rho}}$ with $k$ in place of $\boldsymbol{\nu}$.

In general, it may happen that $H(X)_{\nu}^{\hat{\mu}} \cap H(X)_{k} \neq(0)$ for several $k$ 's, so that $H(X)_{\nu}^{\hat{\mu}}$ does not correspond to a space of holomorphic sections of some power of $A$. Furthermore, $H(X)_{\nu}^{\hat{\mu}}$ may be infinite-dimensional. The latter circumstance does not occur, however, if $\mathbf{0} \notin \Phi(M)$ (see $\S 2$ of [P1]). We shall make the following Basic Assumption (henceforth referred to as BA):

Basic Assumption 1.1. $\Phi$ and $\boldsymbol{\nu}$ satisfy the following properties:

1. $\boldsymbol{\nu} \neq \mathbf{0}$ is coprime, that is, l.c.d. $\left(\nu_{1}, \ldots, \nu_{r}\right)=1$;

2. $\Phi$ is nowhere vanishing, that is, $\mathbf{0} \notin \Phi(M)$;

3. $\Phi$ is transverse to the ray $\mathbb{R}_{+} \cdot \imath \boldsymbol{\nu}$, and $M_{\boldsymbol{\nu}}:=\Phi^{-1}\left(\mathbb{R}_{+} \cdot \imath \boldsymbol{\nu}\right) \neq \emptyset$.

If the previous properties are satisfied, then $\mu^{X}$ is generically locally free [P1]; perhaps after replacing $T$ with its quotient by a finite subgroup, we may and will assume without loss of generality that $\mu^{X}$ is generically free. 
Let us assume that BA holds. Then $H(X)_{k \nu}^{\hat{\mu}}=(0)$ for all $k \leq 0(\S 2$ of [P1]). We are interested in the sequence of spaces of finite-dimensional vector spaces $\left(H(X)_{k \nu}^{\hat{\mu}}\right)_{k=1}^{+\infty}$ associated to the weights on the ray $\mathbb{R}_{+} \cdot \boldsymbol{\nu}$. The corresponding 'equivariant Szegö projectors' $\Pi_{k \nu}^{\hat{\mu}}: L^{2}(X) \rightarrow H(X)_{k \nu}^{\hat{\mu}}$ are smoothing operators (that is, they have $\mathcal{C}^{\infty}$ integral kernels). Furthemore, $M_{\nu} \subseteq M$ is a $T$-invariant coisotropic connected compact submanifold of real codimension $r-1$ [P1]. The local and global asymptotics for $k \rightarrow+\infty$ of the integral kernels $\Pi_{k \nu}^{\hat{\mu}}$ and their concentration behaviour along $M_{\nu}$ were studied in [P1], P2], and related variants in the presense of additional symmetries where investigated in $\mathrm{Ca}$.

Our present aim is to clarify the geometric significance of the sequence $\left(H(X)_{k \nu}^{\hat{\mu}}\right)_{k=1}^{+\infty}$, generalizing the interpretation of the sequence $\left(H(X)_{k}\right)$ in terms of the spaces $H^{0}\left(M, A^{\otimes k}\right)$. We shall prove the following:

Theorem 1.1. Assume BA holds. Then there exists a $(d+1-r)$-dimensional connected compact complex orbifold $N_{\nu}$, and a positive holomorphic orbifold line bundle $B_{\boldsymbol{\nu}}$ on $N_{\boldsymbol{\nu}}$, naturally constructed from $A, \boldsymbol{\nu}$ and $\Phi$, such that the following holds:

1. for $k \geq 1$, there is a natural injection $\delta_{k}: H(X)_{k \nu}^{\hat{\mu}} \hookrightarrow H^{0}\left(N_{\nu}, B_{\nu}^{\otimes k}\right)$;

2. $\delta_{k}$ is an isomorphism if $k \gg 0$.

Corollary 1.1. If $k \gg 0$,

$$
\operatorname{dim} H(X)_{k \boldsymbol{\nu}}^{\hat{\mu}}=\chi\left(N_{\nu}, B_{\nu}^{\otimes k}\right) .
$$

Obviously with no pretense of exhaustiveness, discussions of orbifolds and orbifold line bundles (also known as $V$-manifolds and line $V$-bundles) can be found in [S1], [S2], [B], [Ka], ALR], [BG]; specific treatments of Hamiltonian actions on symplectic orbifolds can be found in [LT] and [MS].

The geometric significance of the Theorem lies in the relation between the polarized orbifold $\left(N_{\boldsymbol{\nu}}, B_{\boldsymbol{\nu}}\right)$ and the 'prequantum data' $(A, \Phi, \boldsymbol{\nu})$. It is therefore in order to outline how the former is constructed from the latter. The following statements will be clarified and proved in $\$ 2$.

Let $\tilde{T} \cong\left(\mathbb{C}^{*}\right)^{r}$ be the complexification of $T$. Then $\mu^{X}$ extends to an holomorphic line bundle action $\tilde{\mu}^{A^{\vee}}: \tilde{T} \times A^{\vee} \rightarrow A^{\vee}$. Let $A_{0}^{\vee}$ be the complement of the zero section in $A^{\vee}$, and let $A_{\nu}^{\vee} \subset A_{0}^{\vee}$ be the inverse image of $M_{\nu}$. Let $\tilde{A}_{\nu}^{\vee}:=\tilde{T} \cdot A_{\nu}^{\vee}$ be its saturation under $\tilde{\mu}^{A^{\vee}}$.

Then $\tilde{\mu}^{A^{\vee}}$ is proper and locally free on $\tilde{A}_{\nu}^{\vee}$, and $N_{\nu}=\tilde{A}_{\nu}^{\vee} / \tilde{T}$. Thus the projection $p_{\boldsymbol{\nu}}: \tilde{A}_{\nu}^{\vee} \rightarrow N_{\boldsymbol{\nu}}$ is a principal $V$-bundle with structure group $\tilde{T}$ over $N_{\nu}[\mathrm{S} 2]$. 
Furthermore, $\chi_{\nu}: T \rightarrow S^{1}$ extends to a character $\tilde{\chi}_{\nu}: \tilde{T} \rightarrow \mathbb{C}^{*}$; the datum of $p_{\boldsymbol{\nu}}$ and $\tilde{\chi}_{\boldsymbol{\nu}}$ determines the orbifold line bundle $B_{\boldsymbol{\nu}}$. Similarly, $B_{\boldsymbol{\nu}}^{\otimes k}$ (or $B_{k \boldsymbol{\nu}}$ ) denotes the orbifold line bundle associated to $p_{\boldsymbol{\nu}}$ and $\tilde{\chi}_{k \boldsymbol{\nu}}=\tilde{\chi}_{\boldsymbol{\nu}}^{k}$.

We can give the following alternative algebro-geometric characterization of $\tilde{A}_{\boldsymbol{\nu}}^{\vee}$. Let $\boldsymbol{\nu}^{\perp} \subset \mathbb{R}^{r}$ be the orthocomplement of $\boldsymbol{\nu}$ with respect to the standard scalar product, and consider the (Abelian) subalgebra $\imath \boldsymbol{\nu}^{\perp} \leqslant \mathfrak{t}$. Let $T_{\nu^{\perp}}^{r-1} \leqslant T$ be the corresponding subtorus, $\tilde{T}_{\boldsymbol{\nu}^{\perp}}^{r-1} \leqslant \tilde{T}$ be its complexification. The restriction of $\tilde{\mu}^{M}$ to $\tilde{T}_{\boldsymbol{\nu}^{\perp}}^{r-1}$ is an holomorphic action $\tilde{\gamma}^{M}$ of $\tilde{T}_{\boldsymbol{\nu}^{\perp}}^{r-1}$ on $(M, J)$, with a built-in complex linearization $\tilde{\gamma}^{A^{\vee}}: \tilde{T}_{\nu^{\perp}}^{r-1} \times A^{\vee} \rightarrow A^{\vee}$. Let $\tilde{M}_{\nu} \subseteq M$ be the locus of (semi)stable points of $\tilde{\gamma}^{M}$; then $\tilde{A}_{\nu}^{\vee}$ is the inverse image of $\tilde{M}_{\nu}$ in $A_{0}^{\vee}$.

Up to a natural isomorphism, an alternative description of $N_{\nu}$ is as follows. Let $X_{\nu}:=\pi^{-1}\left(M_{\nu}\right)$. Then $T$ acts locally freely on $X_{\nu}$, and $N_{\nu} \cong X_{\nu} / T$. This description is instrumental in describing the positivity of $B_{\boldsymbol{\nu}}$ and the Kähler structure of $N_{\boldsymbol{\nu}}$.

When $r=1, M=\mathbb{P}^{d}$, and $A$ is the hyperplane line bundle with the standard metric, we have $X_{\nu}=X=S^{2 d+1}$; thus the previous construction generalizes the one of weighted projective spaces (see also the discussions in [P2] and [P3]).

\section{Preliminaries}

This section is devoted to a closer description of the geometric setting, and to the statement and proof of a series of geometric results that will combine into the proof of Theorem 1.1.

Notation 2.1. We shall adopt the following notation and conventions.

1. If a Lie group $G$ with Lie algebra $\mathfrak{g}$ acts smoothly on a manifold $R$, for any $\xi \in \mathfrak{g}$ we shall denote by $\xi_{R} \in \mathfrak{X}(R)$ the vector field on $R$ generated by $\xi$.

2. If $r \in R$ and $\mathfrak{l} \subseteq \mathfrak{g}$ is a vector subspace, we shall set

$$
\mathfrak{l}_{R}(r):=\left\{\xi_{R}(r): \xi \in \mathfrak{l}\right\} \subseteq T_{r} R .
$$

3. Given an isomorphism $T \cong\left(S^{1}\right)^{r}$, we have $\mathfrak{t} \cong \imath \mathbb{R}^{r}$. If we identify the Lie algebra $\tilde{\mathfrak{t}}$ of $\tilde{T} \cong \mathbb{R}_{+}^{r} \times T$ with $\mathbb{C}^{r} \cong \mathbb{R}^{r} \oplus \imath \mathbb{R}^{r}, \mathfrak{t}$ corresponds to the imaginary summand $\imath \mathbb{R}^{r}$. For $\mathbf{x}=\left(\begin{array}{lll}x_{1} & \cdots & x_{r}\end{array}\right) \in \mathbb{R}^{r}$, we have $e^{\mathbf{x}}:=\left(\begin{array}{lll}e^{x_{1}} & \cdots & e^{x_{r}}\end{array}\right) \in \mathbb{R}_{+}^{r} \leqslant \tilde{T}^{r}$, while $e^{\imath \mathbf{x}}:=\left(\begin{array}{lll}e^{\imath x_{1}} & \cdots & e^{\imath x_{r}}\end{array}\right) \in T^{r}$. 
4. We shall equivariantly identify $\mathfrak{t} \cong \mathfrak{t}^{\vee}$, and view $\Phi$ as $\mathfrak{t}$-valued.

5. If $V$ is any Euclidean vector space and $\epsilon>0, V(\epsilon) \subset V$ will denote the open ball in $V$ centered at the origin and of radius $\epsilon$.

6. $g(\cdot, \cdot):=\omega(\cdot, J(\cdot))$ is the Riemannian metric associated to $\omega$.

7. $J^{\prime}$ is the complex structure of $A^{\vee}$.

8. The superscript $\sharp$ will denote horizontal lifts from $M$ to either $X$ or $A^{\vee}$, according to the context, and will be applied to both tangent vectors and vector subspaces of tangent spaces.

9. $\pi: X \rightarrow M$ and $\pi^{\prime}: A_{0}^{\vee} \rightarrow M$ are the projections.

10. If $\beta^{Z}: G \times Z \rightarrow Z$ is an action of the group $G$ on the set $Z$, and if $S \subseteq Z$ is $G$-invariant, we shall often denote by $\beta^{S}: G \times S \rightarrow S$ the restricted action. Thus, for example, $\tilde{T}$ acts on $A^{\vee}$ by $\tilde{\mu}^{A^{\vee}}$, on $A_{0}^{\vee} \subset A^{\vee}$ by $\tilde{\mu}^{A_{0}^{\vee}}$, on $\tilde{A}_{\nu}^{\vee} \subseteq A_{0}^{\vee}$ by $\tilde{\mu}^{\tilde{A}_{\nu}^{\vee}}$.

\subsection{The locus $M_{\nu} \subseteq M$}

Let $\gamma^{M}: T_{\nu^{\perp}}^{r-1} \times M \rightarrow M$ be the action induced by restriction of $\mu^{M}$. Then $\gamma^{M}$ is Hamiltonian with respect to $2 \omega$, and its moment map $\Phi_{\boldsymbol{\nu}^{\perp}}: M \rightarrow \imath \boldsymbol{\nu}^{\perp}$ is the composition of $\Phi$ with the orthogonal projection $\mathfrak{t} \rightarrow \imath \boldsymbol{\nu}^{\perp}$. Assuming $\mathrm{BA}$, we can draw the following conclusions:

1. $\mathbf{0} \in \imath \boldsymbol{\nu}^{\perp}$ is a regular value of $\Phi_{\boldsymbol{\nu}^{\perp}}$;

2. $M_{\boldsymbol{\nu}}=\Phi_{\boldsymbol{\nu}^{\perp}}^{-1}(\mathbf{0})$ is a compact and connected coisotropic submanifold of $M$, of (real) codimension $r-1$;

3. $\gamma^{M}$ is locally free along $M_{\boldsymbol{\nu}^{\perp}}$, that is,

$$
\operatorname{dim}\left(\imath \boldsymbol{\nu}^{\perp}\right)_{M}(m)=r-1 \quad \forall m \in M_{\boldsymbol{\nu}^{\perp}} ;
$$

4. for every $m \in M_{\boldsymbol{\nu}}$, we have

$$
T_{m} M_{\boldsymbol{\nu}}=\left(\imath \boldsymbol{\nu}^{\perp}\right)_{M}(m)^{\perp^{\omega_{m}}}=J_{m}\left(\left(\iota \boldsymbol{\nu}^{\perp}\right)_{M}(m)\right)^{\perp_{g_{m}}} .
$$

This implies the following statement. Let us define

$$
\Psi:(\mathbf{x}, m) \in\left(\imath \boldsymbol{\nu}^{\perp}\right) \times M_{\boldsymbol{\nu}} \mapsto \tilde{\mu}_{e^{\mathbf{x}}}(m) \in M .
$$


Lemma 2.1. Given Basic Assumption 1.1, the following holds:

1. $\tilde{\gamma}^{M}$ is locally free along $M_{\boldsymbol{\nu}}$;

2. for any sufficiently small $\epsilon>0, \Psi$ in (7) restricts to a diffeomorphism between $\left(\iota \boldsymbol{\nu}^{\perp}\right)(\epsilon) \times M_{\boldsymbol{\nu}}$ and an open tubular neighborhood $U_{\epsilon}$ of $M_{\boldsymbol{\nu}}$ in $M$.

\subsection{The locus $X_{\nu} \subseteq X$ and its saturation $\tilde{X}_{\nu}$ in $A^{\vee}$}

Let us set:

$$
X_{\nu}:=\pi^{-1}\left(M_{\nu}\right) \subseteq X
$$

If $x \in X_{\nu}$, then in view of (41)

$$
\left(\imath \boldsymbol{\nu}^{\perp}\right)_{X}(x)=\left(\iota \boldsymbol{\nu}^{\perp}\right)_{M}(m)^{\sharp} .
$$

We have the following analogue of Lemma 2.1 .

Lemma 2.2. Given BA 1.1, the following holds:

1. $\mu^{X}$ is locally free along $X_{\nu}$;

2. for any $x \in X_{\boldsymbol{\nu}}$,

$$
T_{x} X_{\nu} \cap J_{x}^{\prime}\left(\mathfrak{t}_{A \vee}(x)\right)=(0) ;
$$

3. for all suitably small $\epsilon>0$, the map

$$
\Psi^{\prime}:(\mathbf{x}, x) \in(\imath \mathfrak{t}) \times X_{\nu} \mapsto \tilde{\mu}_{e^{\mathbf{x}}}^{A^{\vee}}(x) \in A_{0}^{\vee}
$$

determines a diffemorphism from $(\imath \mathfrak{t})(\epsilon) \times X_{\nu}$ to a tubular neighborhood of $X_{\nu}$ in $A^{\vee}$;

4. $\tilde{\mu}^{A^{\vee}}$ is locally free along $X_{\nu}$.

Proof of Lemma 2.2. That $\mu^{X}$ is locally free on $X_{\nu}$ under the transversality assumption in BA is proved in $\S 2$ of [P1].

By the discussion in 92.1 , if $x \in X_{\nu}$ and $m=\pi(x)$ then

$$
J_{m}\left(\iota \nu^{\perp}\right)_{M}(m)^{\sharp} \subseteq T_{x} X
$$

is the normal space of $X_{\nu}$ in $X$ at $x$; hence given (9) we have

$$
T_{x} X_{\boldsymbol{\nu}} \cap J_{x}^{\prime}\left(\imath \boldsymbol{\nu}^{\perp}\right)_{X}(x)=T_{x} X_{\boldsymbol{\nu}} \cap J_{m}\left(\iota \boldsymbol{\nu}^{\perp}\right)_{M}(m)^{\sharp}=(0) .
$$


Furthermore, by definition of $X_{\nu}$ there exists a smooth function $\lambda_{\nu}: M_{\nu} \rightarrow$ $\mathbb{R}_{+}$such that

$$
(\imath \boldsymbol{\nu})_{X}(x)=(\imath \boldsymbol{\nu})_{M}(m)^{\sharp}-\left.\lambda_{\boldsymbol{\nu}}(m)\|\boldsymbol{\nu}\|^{2} \partial_{\theta}\right|_{x} \notin \operatorname{Hor}(X)_{x}, \quad \forall x \in X_{\boldsymbol{\nu}} .
$$

If $r$ denotes the radial coordinate along the fibers of $A^{\vee}$, this implies

$$
J_{x}^{\prime}\left((\imath \boldsymbol{\nu})_{X}(x)\right)=J_{m}\left((\imath \boldsymbol{\nu})_{M}(m)\right)^{\sharp}+\left.\lambda_{\boldsymbol{\nu}}(m)\|\boldsymbol{\nu}\|^{2} \partial_{r}\right|_{x} \in T_{x} A^{\vee} \backslash T_{x} X .
$$

The second statement follows from (10), (11) and (12).

The third statement is an immediate consequence of the second.

Since $X_{\boldsymbol{\nu}}$ is a $T$-invariant submanifold of $A^{\vee},\left(\imath \boldsymbol{\nu}^{\perp}\right)_{X}(x) \subseteq T_{x} X_{\boldsymbol{\nu}}$ for any $x \in X_{\nu}$. Hence if $x \in X_{\boldsymbol{\nu}}$ and $m=\pi(x)$ then by (10)

$$
\left(\imath \boldsymbol{\nu}^{\perp}\right)_{X}(x) \cap J_{x}^{\prime}\left(\imath \boldsymbol{\nu}^{\perp}\right)_{X}(x)=\left(\imath \boldsymbol{\nu}^{\perp}\right)_{M}(m)^{\sharp} \cap J_{m}\left(\imath \boldsymbol{\nu}^{\perp}\right)_{M}(m)^{\sharp}=(0) .
$$

Together with the first statement, this implies $\operatorname{dim}_{\mathbb{C}}\left(\tilde{\mathfrak{t}}_{A} \vee(x)\right)=r$ for any $x \in X_{\nu}$.

Let us consider the saturation

$$
\tilde{X}_{\nu}:=\tilde{T} \cdot X_{\nu} \subseteq A^{\vee}
$$

Corollary 2.1. $\tilde{X}_{\nu}$ is open in $A^{\vee}$.

Corollary 2.2. If Basic Assumption 1.1 holds, then $\mu^{X}$ is generically free on $X_{\nu}$.

Proof of Corollary 2.2. If the general $x \in X_{\nu}$ had non-trivial stabilizer in $T$, the same would hold of the general $\ell \in \tilde{X}_{\boldsymbol{\nu}}$; since the latter is open in $A_{0}^{\vee}$, this contradicts the assumption that $\mu^{A^{\vee}}$ is generically free.

Corollary 2.3. If $B A$ 1.1 holds, then $N_{\nu}^{\prime}:=X_{\nu} / T$ is a compact orbifold of real dimension $2(d+1-r)$, and the projection

$$
p_{\nu}^{\prime}: X_{\nu} \rightarrow N_{\nu}^{\prime}
$$

is a principal $V$-bundle with structure group $T$.

Remark 2.1. Associated to $p_{\nu}^{\prime}$ and the character $\chi_{\nu}$ there is a orbifold complex line bundle $B_{\nu}^{\prime}$ on $N_{\nu}^{\prime}$. 


\subsection{The Kähler structure of $A_{0}^{\vee}$}

Let $\varrho: A_{0}^{\vee} \rightarrow \mathbb{R}$ denote the square norm function in the Hermitian metric $h$, and set

$$
\tilde{\omega}:=2 \mathrm{~d}\left(\Im\left(\partial \varrho^{1 / 2}\right)\right)=2 \imath \partial \bar{\partial}\left(\varrho^{1 / 2}\right) .
$$

If $\pi^{\prime}: A_{0}^{\vee} \rightarrow M$ is the projection, then

$$
\tilde{\omega}=2 \varrho^{1 / 2} \pi^{\prime *}(\omega)+\frac{\imath}{2 \varrho^{3 / 2}} \partial \varrho \wedge \bar{\partial} \varrho .
$$

The contact action $\mu^{X}: T \times X \rightarrow X$ extends to an holomorphic unitary action $\mu^{A^{\vee}}: T \times A_{0}^{\vee} \rightarrow A_{0}^{\vee}$.

Proposition 2.1. $\tilde{\omega}$ is a $\mu^{A^{\vee}}$-invariant exact Kähler form on $A_{0}^{\vee}$.

Proof. Since $\mu^{A^{\vee}}$ preserves both $\varrho$ and the complex structure, by its definition $\tilde{\omega}$ is a $\mu^{A^{\vee}}$-invariant closed $(1,1)$-form. Thus we need only prove that $\tilde{\omega}$ is non-degenerate.

The unique connection compatible with both $h$ and the holomorphic structure determines an invariant decomposition

$$
T A_{0}^{\vee}=\operatorname{Hor}\left(A_{0}^{\vee}\right) \oplus \operatorname{Ver}\left(A_{0}^{\vee}\right),
$$

where

$$
\operatorname{Hor}\left(A_{0}^{\vee}\right):=\operatorname{ker}(\partial \varrho), \quad \operatorname{Ver}\left(A_{0}^{\vee}\right):=\operatorname{ker}\left(\mathrm{d} \pi^{\prime}\right) \subset T A^{\vee}
$$

denote the horizontal and vertical tangent bundles. Then $\operatorname{Hor}\left(A_{0}^{\vee}\right)$ and $\operatorname{Ver}\left(A_{0}^{\vee}\right)$ are complex vector subbundles of $T A_{0}^{\vee}$, and by (17) they are orthogonal for $\tilde{\omega}$. Furthermore, the first summand on the right hand side of (17) is symplectic on $\operatorname{Hor}\left(A_{0}^{\vee}\right)$ and vanishes on $\operatorname{Ver}\left(A_{0}^{\vee}\right)$, and conversely for the second summand. Hence $\tilde{\omega}$ is non-degenerate.

Corollary 2.4. $\mu^{A^{\vee}}$ is Hamiltonian on $\left(A_{0}^{\vee}, \tilde{\omega}\right)$, with moment map

$$
\tilde{\Phi}:=\varrho^{1 / 2} \cdot \Phi \circ \pi^{\prime}: A_{0}^{\vee} \rightarrow \mathfrak{t},
$$

where $\pi^{\prime}: A_{0}^{\vee} \rightarrow M$ is the projection.

Proof of Corollary 2.4. Given an exact symplectic manifold $(R, \eta)$ with $\eta=$ $-\mathrm{d} \lambda$, and a smooth Lie group action $\varsigma: G \times N \rightarrow N$ preserving $\lambda$, it is wellknown that $\varsigma$ is Hamiltonian, with moment map $\Upsilon: R \rightarrow \mathfrak{g}^{\vee}$ determined by the relation

$$
v^{\xi}:=\langle\Upsilon, \xi\rangle=\iota\left(\xi_{R}\right) \lambda \in \mathcal{C}^{\infty}(M)
$$


In our setting, $R=A_{0}^{\vee}, \varsigma=\mu^{A^{\vee}}, \eta=\tilde{\omega}$ and, in view of (16),

$$
\lambda=-2\left(\Im\left(\partial \varrho^{1 / 2}\right)\right)=\imath\left(\partial \varrho^{1 / 2}-\bar{\partial} \varrho^{1 / 2}\right) ;
$$

furthermore, for any $\xi \in \mathfrak{t}$ we have

$$
\xi_{A^{\vee}}=\xi_{M}^{\sharp}-\left\langle\Phi \circ \pi^{\prime}, \xi\right\rangle \partial_{\vartheta} .
$$

Since $\xi_{M}^{\sharp}$ is a section of $\operatorname{Hor}\left(A_{0}^{\vee}\right)$, it follows from (19) that $\iota\left(\xi_{M}^{\sharp}\right) \partial \varrho=0$. Furthermore, one can verify that $\imath\left(\partial_{\theta}\right) \partial \rho=\imath \rho$. Putting this together, we conclude that $\mu^{A^{\vee}}$ is Hamiltonian, and furthermore the component $\tilde{\phi}^{\xi}=$ $\langle\tilde{\Phi}, \xi\rangle$ of the moment map is

$$
\begin{aligned}
\tilde{\phi}^{\xi} & =\imath \cdot \varrho^{-1 / 2} \iota\left(\xi_{M}^{\sharp}-\left(\varphi^{\xi} \circ \pi^{\prime}\right) \partial_{\theta}\right)(\partial \varrho-\bar{\partial} \varrho) \\
& =\left(\varphi^{\xi} \circ \pi^{\prime}\right) \varrho^{1 / 2} .
\end{aligned}
$$

Let $\{\cdot, \cdot\}_{A_{0}^{\vee}}$ denote by Poisson brackets on $\left(A_{0}^{\vee}, \tilde{\omega}\right)$. Since $\mu^{A^{\vee}}$ is unitary, in view of (20) we conclude the the following.

Corollary 2.5. $\left\{\tilde{\phi}^{\xi}, \tilde{\phi}^{\eta}\right\}$ vanishes, $\forall \xi, \eta \in \mathfrak{t}$. In particular, the orbits of $\mu^{A^{\vee}}$ in $A_{0}^{\vee}$ are isotropic for $\tilde{\omega}$.

Therefore:

Corollary 2.6. For every $\ell \in A_{0}^{\vee}, \mathfrak{t}_{A^{\vee}}(\ell) \subseteq T_{\ell} A^{\vee}$ is totally real, that is,

$$
\mathfrak{t}_{A \vee}(\ell) \cap J_{\ell}^{\prime}\left(\mathfrak{t}_{A^{\vee}}(\ell)\right)=(0) .
$$

By Proposition 1.6 and Theorem 1.12 in [Sj], Corollary 2.4 has the following consequences.

Corollary 2.7. For every $\ell \in A_{0}^{\vee}$, the following holds:

1. the stabilizer $\tilde{T}_{\ell} \leqslant \tilde{T}$ of $\ell$ for $\tilde{\mu}^{A^{\vee}}$ is the complexification of of the stabilizer $T_{\ell} \leqslant T$ of $\ell$ for $\mu^{A^{\vee}}$;

2. there exists an holomorphic slice for $\tilde{\mu}^{A_{0}^{\vee}}$ at $\ell$.

Let $A_{l f}^{\vee} \subseteq A_{0}^{\vee}$ be the open subset where $\tilde{\mu}^{A_{0}^{\vee}}$ is locally free. It follows from Proposition 1.6 of $[\mathrm{Sj}]$ and Corollary 2.5 above that the stabilizer in $\tilde{T}$ of any $\ell \in A_{l f}^{\vee}$ is finite and contained in $T$. 
Definition 2.1. Following [Sj], we shall call $\tilde{\mu}^{A_{0}^{\vee}}$ proper at $\ell \in A_{0}^{\vee}$ if for all sequences $\left(\ell_{j}\right) \subset A_{0}^{\vee}$ and $\left(\tilde{t}_{j}\right) \subset \tilde{T}$ such that $\ell_{j} \rightarrow \ell$ and $\tilde{\mu}_{\tilde{t}_{j}}^{A_{0}^{\vee}}\left(\ell_{j}\right)$ converges to some point in $A_{0}^{\vee},\left(\tilde{t}_{j}\right)$ is convergent in $\tilde{T}$.

Remark 2.2. Let $U \subseteq A_{0}^{\vee}$ be a $\tilde{T}$-invariant open set. Then:

1. if $\tilde{\mu}^{A_{0}^{\vee}}$ is proper at every $\ell \in U$, then so is a fortiori $\tilde{\mu}^{U}$;

2. if $\tilde{\mu}^{U}$ is proper at every $\ell \in U, \tilde{\mu}^{U}$ is (globally) proper.

Corollary 2.8. Given BA, the following holds:

1. $\tilde{\mu}^{A_{0}^{\vee}}$ is proper on $A_{l f}^{\vee}$ (that is, $\tilde{\mu}^{A_{l f}^{\vee}}$ is proper);

2. $\tilde{X}_{\nu} \subseteq A_{l f}^{\vee}$;

3. $\tilde{\mu}^{A_{0}^{\vee}}$ is proper on $\tilde{X}_{\nu}$ (that is, $\tilde{\mu}^{\tilde{X}_{\nu}}$ is proper).

Proof of Corollary 2.8. We have remarked that the stabilizer in $\tilde{T}$ of any $\ell \in A_{l f}^{\vee}$ coincides with the stabilizer of $\ell$ in $T$, and therefore it is finite. In view of Theorem 1.22 of $[\mathrm{Sj}], \tilde{\mu}^{A_{0}^{\vee}}$ is proper at any $\ell \in A_{l f}^{\vee}$, and therefore it is proper on $A_{l f}^{\vee}$. This proves the first statement.

We know that $\mu^{X_{\nu}}$ is locally free; in other words, $\mu^{A_{0}^{\vee}}$ is locally free along $X_{\boldsymbol{\nu}}$. Therefore, $\mu^{A_{0}^{\vee}}$ is locally free on $\tilde{X}_{\boldsymbol{\nu}}=\tilde{T} \cdot X_{\boldsymbol{\nu}}$, because $\tilde{T}$ is Abelian. Therefore, by Corollary 2.7, the stabilizer of any $\ell \in \tilde{X}_{\nu}$ in $\tilde{T}$ for $\tilde{\mu}^{A_{0}^{\vee}}$ is finite, since it coincides with the stabilizermof $\ell$ in $T$ for $\mu^{A_{0}^{\vee}}$. This proves the second statement.

The third statement is a straighforward consequence of the first two.

The structure $S^{1}$-action $\rho^{X}$ extends to the holomorphic action

$$
\tilde{\rho}^{A^{\vee}}:(z, \ell) \in \mathbb{C}^{*} \times A_{0}^{\vee} \mapsto z^{-1} \ell \in A_{0}^{\vee},
$$

whose orbits are the fibers of $A_{0}^{\vee}$ over $M$.

Lemma 2.3. $\tilde{X}_{\nu}$ is $\tilde{\rho}^{A^{\vee}}$-invariant.

Proof of Lemma 2.3. Since $\tilde{\mu}^{A^{\vee}}$ and $\tilde{\rho}^{A^{\vee}}$ commute, it suffices to show that for any $x \in X_{\nu}$ and $z \in \mathbb{C}^{*}$ we have $z x \in \tilde{X}_{\nu}$.

Let us set

$$
C_{x}:=\left\{z \in \mathbb{C}^{*}: z x \in \tilde{X}_{\nu}\right\} .
$$

Then $1 \in C_{x}$ and $C_{x}$ is open in $\mathbb{C}^{*}$ because scalar multiplication is continuous and $\tilde{X}_{\nu}$ is open in $A^{\vee}$ (Corollary 2.1). 
Suppose $z_{\infty} \in \mathbb{C}^{*}$ is a limit point of $C_{x}$. Then there exist $z_{1}, z_{2}, \ldots \in C_{x}$ such that $z_{i} \rightarrow z_{\infty}$. By definition of $C_{x}$, for any $i=1,2, \ldots$ we have $z_{i} x \in \tilde{X}_{\nu}$ for any $i=1,2, \ldots$. By definition of $\tilde{X}_{\boldsymbol{\nu}}$, therefore, there exist $\tilde{t}_{i} \in \tilde{T}$ and $x_{i} \in X_{\nu}$ such that $z_{i} x=\tilde{\mu}_{\tilde{t}_{i}}^{A^{\vee}}\left(x_{i}\right)$. Since $A_{l f}^{\vee}$ in Corollary 2.8 is clearly $\mathbb{C}^{*}$-invariant, we have $z_{\infty} x \in A_{l f}^{\vee}$. Thus

$$
\tilde{\mu}_{\tilde{t}_{i}}^{A^{\vee}}\left(x_{i}\right)=z_{i} x \rightarrow z_{\infty} x \in A_{l f}^{\vee}
$$

By Corollary 2.8 and the compactness of $X_{\boldsymbol{\nu}}$, perhaps replacing $\left(\tilde{t}_{i}\right)$ and $\left(x_{i}\right)$ by subsequences, we may assume that $\tilde{t}_{i} \rightarrow \tilde{t}_{\infty} \in \tilde{T}$ and $x_{i} \rightarrow x_{\infty} \in X_{\boldsymbol{\nu}}$. Hence

$$
z_{\infty} x=\tilde{\mu}_{\tilde{t}_{\infty}^{\vee}}^{A^{\vee}}\left(x_{\infty}\right) \in \tilde{X}_{\nu} \Rightarrow z_{\infty} \in C_{x} .
$$

We conclude that $C_{x}=\mathbb{C}^{*}$ for any $x \in X_{\nu}$.

Let us set, as in the Introduction,

$$
A_{\nu}^{\vee}:=\left(\pi^{\prime}\right)^{-1}\left(M_{\nu}\right), \quad \tilde{A}_{\nu}^{\vee}:=\tilde{T} \cdot A_{\nu}^{\vee}
$$

Corollary 2.9. $\tilde{X}_{\nu}=\tilde{A}_{\nu}^{\vee}$.

Proof of Corollary 2.9. Since $X_{\nu} \subset A_{\nu}^{\vee}$, clearly $\tilde{X}_{\nu} \subseteq \tilde{A}_{\nu}^{\vee}$. On the other hand, $\tilde{X}_{\nu}$ is $\mathbb{C}^{*}$-invariant by Lemma 2.3 and contains $X_{\nu}$, hence $\tilde{X}_{\nu} \supseteq A_{\nu}$. Since $\tilde{X}_{\nu}$ is $\tilde{T}$-invariant, we also have $\tilde{X}_{\nu} \supseteq \tilde{A}_{\nu}^{\vee}$.

It follows from Lemma 2.3 that $\tilde{X}_{\nu}$ is the inverse image of a $\tilde{T}$-invariant open set of $M$. More precisely, let

$$
M_{\nu}^{\prime}:=\tilde{T} \cdot M_{\nu} \subseteq M .
$$

Since $\pi^{\prime}$ is a submersion and intertwines $\tilde{\mu}^{M}$ and $\tilde{\mu}^{A^{\vee}}$, we conclude the following:

Corollary 2.10. $M_{\nu}^{\prime}$ is open in $M$ and $\tilde{X}_{\nu}=\left(\pi^{\prime}\right)^{-1}\left(M_{\nu}^{\prime}\right)$.

As in the Introduction, let $\tilde{M}_{\nu} \subseteq M$ be the dense open subset of stable points for $\gamma^{M}$. Obviously $\tilde{M}_{\boldsymbol{\nu}}$ is $\tilde{\gamma}^{M}$-invariant (notation is as in the Introduction and 92.1 ).

Lemma 2.4. $\tilde{M}_{\nu}=M_{\nu}^{\prime}$.

Proof of Lemma 2.4. Since $\mathbf{0} \in \imath \boldsymbol{\nu}^{\perp}$ is a regular value of $\Phi_{\boldsymbol{\nu}^{\perp}}, \tilde{M}_{\boldsymbol{\nu}}=\tilde{T}_{\boldsymbol{\nu}^{\perp}}^{r-1}$. $M_{\boldsymbol{\nu}}$. Hence trivially $\tilde{M}_{\boldsymbol{\nu}}=\tilde{T}_{\boldsymbol{\nu}^{\perp}}^{r-1} \cdot M_{\boldsymbol{\nu}} \subseteq \tilde{T} \cdot M_{\boldsymbol{\nu}}=M_{\boldsymbol{\nu}}^{\prime}$.

To prove the converse inclusion it suffices to check that $\tilde{M}_{\boldsymbol{\nu}}$ is $\tilde{T}$-invariant. For $k=1,2, \ldots$, let $\tilde{\hat{\mu}}^{(k)}$ be the representation of $\tilde{T}$ on $H^{0}\left(M, A^{\otimes k}\right)$ induced 
by $\tilde{\mu}^{A^{\vee}}$, and let $H^{0}\left(M, A^{\otimes k}\right)^{T^{\nu}{ }^{\perp-1}} \subseteq H^{0}\left(M, A^{\otimes k}\right)$ be the subspace of those sections that are invariant under $\bar{T}_{\boldsymbol{\nu}^{\perp}}^{r-1}$ (equivalently, $\tilde{T}_{\boldsymbol{\nu}^{\perp}}^{r-1}$ ). Then $m \in \tilde{M}_{\boldsymbol{\nu}}$ if and only if for some $k=1,2, \ldots$ there exists $\sigma \in H^{0}\left(M, A^{\otimes k}\right)^{T^{r-1}}$ such that $\sigma(m) \neq 0$. Since $\tilde{T}$ is Abelian, $\tilde{\hat{\mu}}_{\tilde{t}}^{(k)}(\sigma) \in H^{0}\left(M, A^{\otimes k}\right)^{T^{r}{ }^{\perp}}$ for any $\tilde{t} \in \tilde{T}$; therefore, if $m \in M$ is stable for $\gamma^{M}$, then so is $\tilde{\mu}_{\tilde{t}}^{M}(m)$, for any $\tilde{t} \in \tilde{T}$.

In the following, we shall write $\tilde{A}_{\nu}^{\vee}$ for $\tilde{X}_{\nu}$. Since $\tilde{\mu}^{A^{\vee}}$ is holomorphic, proper, effective and locally free on $\tilde{A}_{\nu}^{\vee}$, we reach the following conclusion.

Corollary 2.11. If $B A$ 1.1 holds, then $N_{\nu}:=\tilde{A}_{\nu}^{\vee} / \tilde{T}$ is a compact and connected orbifold of complex dimension $d+1-r$, and the projection

$$
p_{\nu}: \tilde{A}_{\nu}^{\vee} \rightarrow N_{\nu}
$$

is a principal $V$-bundle with structure group $\tilde{T}$.

Proof. Since $\tilde{T}$ acts properly, holomorphically and locally freely on $\tilde{X}_{\boldsymbol{\nu}}, N_{\boldsymbol{\nu}}$ is a connected complex orbifold of dimension $d+1-r$. Furthermore, by definition of $\tilde{X}_{\boldsymbol{\nu}}, p_{\boldsymbol{\nu}}\left(X_{\boldsymbol{\nu}}\right)=N_{\boldsymbol{\nu}}$. Hence $N_{\boldsymbol{\nu}}$ is compact.

Remark 2.3. The holomorphic slices in Corollary 2.7 provide local uniformizing charts for $N_{\boldsymbol{\nu}}$. Associated to $p_{\boldsymbol{\nu}}$ and the character $\tilde{\chi}_{\boldsymbol{\nu}}$ there is an holomorphic orbifold line bundle $B_{\nu}$ on $N_{\nu}$.

\subsection{The isomorphism between $N_{\nu}^{\prime}$ and $N_{\nu}$}

We shall see that $N_{\nu}^{\prime}$ has a natural complex structure, and that the pairs $\left(N_{\nu}^{\prime}, B_{\nu}^{\prime}\right)$ and $\left(N_{\nu}, B_{\nu}\right)$ in Corollaries 2.3 and 2.11 are naturally isomorphic as complex orbifolds and orbifold line bundles.

If $F \subseteq A_{0}^{\vee}$ is an holomorphic slice for $\tilde{\mu}^{A^{\vee}}$ as in Corollary 2.7, let $J^{F}$ be its complex structure. Then $\left(F, J^{F}\right)$ is a complex submanifold of $\left(A_{0}^{\vee}, J^{\prime}\right)$, and provides a local uniformizing chart for the complex orbifold $N_{\boldsymbol{\nu}}$.

On the other hand, given $x \in X_{\nu}$ let $F \subseteq X_{\nu}$ be a slice at $x$ for the action $\mu^{X_{\nu}}: T \times X_{\nu} \rightarrow X_{\nu}$ induced by $\mu^{X}$. The stabilizer $T_{x} \leqslant T$ of $x$ in $T$ is a finite subgroup of $T$, and by Corollary $2.7 T_{x}=\tilde{T}_{x}$ (the stabilizer in $\tilde{T}$ ).

If $\epsilon>0$, let $F_{\epsilon} \subseteq F$ be the intersection of $F$ with an open ball centered at $x$ and radius $\epsilon$, in the Kähler metric on $A_{0}^{\vee}$ associated to $\tilde{\omega}$ in (17).

The proof of the following will be omitted.

Proposition 2.2. If $\epsilon>0$ is suitably small, $F_{\epsilon}$ is a slice for of $\tilde{\mu}^{A_{0}^{\vee}}$. 
Certainly $F$ is not a complex submanifold of $A_{0}^{\vee}$, and in fact it does not contain any complex submanifold of positive dimension. Nonetheless, there is a natural complex structure $J^{F}$ on it, that may be described as follows.

If $\ell \in \tilde{A}_{\nu}^{\vee}$, the tangent space to the $\tilde{T}$-orbit of $\ell, \tilde{\mathfrak{t}}_{A_{0}^{\vee}}(\ell) \subseteq T_{\ell} A_{0}^{\vee}$, is an $r$-dimensional complex subspace; let $S_{\ell} \subset T_{\ell} A_{0}^{\vee}$ be the orthocomplement of $\tilde{\mathfrak{t}}_{A_{0}^{\vee}}(\ell)$ for the Riemannian metric associated to (17). Thus $S_{\ell}$ is a complex subspace of $T_{\ell} A_{0}^{\vee}$, of dimension $d+1-r$, and we have a smoothly varying direct sum decomposition $T_{\ell} A^{\vee}=\tilde{t}_{A^{\vee}}(\ell) \oplus S_{\ell}$. Globally on $\tilde{A}_{\nu}^{\vee}$, this yields a vector bundle decomposition $T A^{\vee}=\tilde{\mathfrak{t}}_{A^{\vee}} \oplus S$. Projecting along $\tilde{\mathfrak{t}}_{A^{\vee}}$, we obtain a morphism of vector bundles $\Pi: T A^{\vee} \rightarrow S$ (on $A_{\nu}^{\vee}$ ).

Let $F$ by any slice for $\tilde{\mu}^{A_{0}^{\vee}}$ in $\tilde{A}_{\nu}^{\vee}$; in particular, by Proposition $2.2, F$ might be a slice for $\mu^{X_{\nu}}$. At any $\ell \in F$, the restriction of $\Pi_{\ell}$ is an isomorphism of real vector spaces $\Pi_{\ell}^{F}: T_{\ell} F \rightarrow S_{\ell}$. We may define an almost complex structure $J^{F}$ on $F$ by declaring $\Pi_{\ell}^{F}$ to be an isomorphism of complex vector spaces for each $\ell \in F$. If $F$ is an holomorphic slice, $J^{F}$ clearly coincides with the complex structure of $F$ as a submaifold of $A_{0}^{\vee}$.

It is clear that the same $J^{F}$ would be defined, if instead of $S$ one had chosen another complementary complex subundle $S^{\prime}$ to $\tilde{\mathfrak{t}}_{A_{0}^{\vee}}$. The following characterization does not involve the choice of a specific sub-bundle.

Lemma 2.5. If $\ell \in F$ and $v \in T_{\ell} F$, then $J_{\ell}^{F}(v)$ is uniquely determined by the conditions:

- $J_{\ell}^{F}(v) \in T_{\ell} L$;

- $J_{\ell}^{F}(v)-J_{\ell}^{\prime}(v) \in \tilde{\mathfrak{t}}_{A^{\vee}}(\ell)$.

Proof of Lemma 2.5. For $v \in T_{\ell} A^{\vee}$, let $v_{t} \in \tilde{\mathfrak{t}}_{A^{\vee}}(\ell)$ and $v_{s} \in S_{\ell}$ be its components. As both $\tilde{\mathfrak{t}}_{A^{\vee}}(\ell)$ and $S_{\ell}$ are complex subspaces for $J_{\ell}^{\prime}$,

$$
J_{\ell}^{\prime}\left(v_{s}\right)=J_{\ell}^{\prime}(v)_{s}, \quad J_{\ell}^{\prime}\left(v_{t}\right)=J_{\ell}^{\prime}(v)_{t} .
$$

By definition of $J^{F}$ if $v \in T_{\ell} A^{\vee}$ then

$$
J_{\ell}^{F}(v)_{s}=J_{\ell}^{\prime}\left(v_{s}\right)=J_{\ell}^{\prime}(v)_{s} .
$$

Hence,

$$
\left(J_{\ell}^{F}(v)-J_{\ell}^{\prime}(v)\right)_{s}=J_{\ell}^{\prime}(v)_{s}-J_{\ell}^{\prime}(v)_{s}=0 \quad \Rightarrow \quad J_{\ell}^{F}(v)-J_{\ell}^{\prime}(v) \in \tilde{\mathfrak{t}}_{A^{\vee}}(\ell) .
$$

Suppose that $I_{\ell}^{F}: T_{\ell} F \rightarrow T_{\ell} F$ is another operator such that $I_{\ell}^{F}(v)-$ $J_{\ell}^{\prime}(v) \in \tilde{\mathfrak{t}}_{A \vee}(\ell)$ for every $v \in T_{\ell} F$. Then (by definition of slice) $\forall v \in T_{\ell} F$ we have

$$
I_{\ell}^{F}(v)-J_{\ell}^{F}(v)=\left(I_{\ell}^{F}(v)-J_{\ell}^{\prime}(v)\right)-\left(J_{\ell}^{F}(v)-J_{\ell}^{\prime}(v)\right) \in T_{\ell} F \cap \tilde{\mathfrak{t}}_{A^{\vee}}(\ell)=(0) .
$$


Consider two slices $F_{1}, F_{2} \subset \tilde{A}_{\nu}^{\vee}$ for $\tilde{\mu}^{A_{0}^{\vee}}$ such that $p_{\boldsymbol{\nu}}\left(F_{1}\right) \subseteq p_{\nu}\left(F_{2}\right)$. Let $\ell_{j} \in F_{j}$ be such that $p_{\boldsymbol{\nu}}\left(\ell_{1}\right)=p_{\boldsymbol{\nu}}\left(\ell_{2}\right)$. Hence there exists $\tilde{t} \in \tilde{T}$ such that $\ell_{2}=\tilde{\mu}_{\tilde{t}}^{A_{0}^{\vee}}\left(\ell_{1}\right)$. Perhaps after restricting $F_{1}$, we may find a unique $\mathcal{C}^{\infty}$ function $f: F_{1} \rightarrow \tilde{\mathfrak{t}}$, such that $f\left(\ell_{1}\right)=\mathbf{0}$ and $\jmath(\ell):=\tilde{\mu}_{\tilde{t} e^{f(\ell)}}^{A^{\vee}}(\ell) \in F_{2}$, for all $\ell \in F_{1}$. Thus $\jmath: F_{1} \rightarrow F_{2}$ is an injection in the sense of Satake ([S1], [S2]).

Lemma 2.6. $\jmath: F_{1} \rightarrow F_{2}$ is $\left(J^{F_{1}}, J^{F_{2}}\right)$-holomorphic.

Proof of Lemma 2.6. By local uniqueness, it suffices to prove that

$$
\mathrm{d}_{\ell_{1}} \jmath:\left(T_{\ell_{1}} F_{1}, J_{\ell_{1}}^{F_{1}}\right) \rightarrow\left(T_{\ell_{2}} F_{2}, J_{\ell_{2}}^{F_{2}}\right)
$$

is $\mathbb{C}$-linear. If $v \in T_{\ell_{1}} F$, we have

$$
\mathrm{d}_{\ell_{1}} f(v) \in \mathfrak{t}, \quad \mathrm{d}_{\ell_{1}} f(v)_{A^{\vee}} \in \mathfrak{X}\left(A^{\vee}\right), \quad \mathrm{d}_{\ell_{1}} f(v)_{A^{\vee}}\left(\ell_{2}\right) \in \tilde{\mathfrak{t}}_{A^{\vee}}\left(\ell_{2}\right) \subseteq T_{\ell_{2}} A^{\vee},
$$

and

$$
\mathrm{d}_{\ell_{1}} \jmath(v)=\mathrm{d}_{\ell_{1}} f(v)_{A^{\vee}}\left(\ell_{2}\right)+\mathrm{d}_{\ell_{1}} \tilde{\mu}_{\tilde{t}}^{A_{0}^{\vee}}(v) .
$$

If $w, w^{\prime} \in T_{\ell_{2}} A^{\vee}$, we shall write $w \equiv w^{\prime}$ to mean that $w-w^{\prime} \in \tilde{\mathfrak{t}}_{A^{\vee}}\left(\ell_{2}\right)$. By (22) , we have $\mathrm{d}_{\ell_{1}} \jmath(v) \equiv \mathrm{d}_{\ell_{1}} \tilde{\mu}_{\tilde{t}}^{A_{0}^{\vee}}(v)$ for every $v \in T_{\ell_{1}} F_{1}$. Replacing $v$ with $J_{\ell_{1}}^{F_{1}}(v)$, in view of Lemma 2.5 we obtain

$$
\begin{aligned}
\mathrm{d}_{\ell_{1}} \jmath\left(J_{\ell_{1}}^{F_{1}}(v)\right) & \equiv \mathrm{d}_{\ell_{1}} \tilde{\mu}_{\tilde{t}}^{A_{0}^{\vee}}\left(J_{\ell_{1}}^{F_{1}}(v)\right) \equiv \mathrm{d}_{\ell_{1}} \tilde{\mu}_{\tilde{t}}^{A_{0}^{\vee}}\left(J_{\ell_{1}}^{\prime}(v)\right) \\
& =J_{\ell_{2}}^{\prime}\left(\mathrm{d}_{\ell_{1}} \tilde{\mu}_{\tilde{t}}^{A_{0}^{\vee}}(v)\right) \equiv J_{\ell_{2}}^{\prime}\left(\mathrm{d}_{\ell_{1}} \jmath(v)\right) \equiv J_{\ell_{2}}^{F_{2}}\left(\mathrm{~d}_{\ell_{1}} \jmath(v)\right) .
\end{aligned}
$$

The first and the last vector in (23) belong to $T_{\ell_{2}} F_{2}$; hence by Lemma 2.5 $\mathrm{d}_{\ell_{1}} \jmath\left(J_{\ell_{1}}^{F_{1}}(v)\right)=J_{\ell_{2}}^{F_{2}}\left(\mathrm{~d}_{\ell_{1}} \jmath(v)\right)$, for all $v \in T_{\ell_{1}} F_{1}$.

In Lemma 2.6, we may assume by Corollary 2.7 that $F_{2}$, say, is holomorphic; hence Lemma 2.6 implies the following.

Corollary 2.12. For any slice $F \subset \tilde{A}_{\nu}^{\vee}$ for $\tilde{\mu}^{A_{0}^{\vee}}, J^{F}$ is integrable.

We may also take $F=F_{1}=F_{2}$ be a slice at $\ell \in A_{0}^{\vee}$, and consider the self-injections of $F$ induced by the stabilizer $T_{\ell} \leqslant T$ of $\ell$.

Corollary 2.13. If $\ell \in A_{\nu}^{\vee}$ and $F \subset A_{\nu}^{\vee}$ is a slice for $\tilde{\mu}^{A_{0}^{\vee}}$ at $\ell$, then $\tilde{T}_{\ell}$ acts holomorphically on $\left(F, J^{F}\right)$.

If we apply these considerations to the slices $F \subseteq X_{\nu}$ for $\mu^{X_{\nu}}$, we conclude the following. 
Corollary 2.14. The V-manifold $N_{\nu}^{\prime}$ in Corollary 2.3 is complex.

Since every $T$-orbit in $X_{\nu}$ is obviously contained in a unique $\tilde{T}$-orbit in $\tilde{A}_{\nu}$, there is a well-defined map

$$
\psi: T \cdot x \in N_{\nu}^{\prime} \mapsto \tilde{T} \cdot x \in N_{\nu}
$$

Let $J^{N_{\nu}^{\prime}}$ and $J^{N_{\nu}}$ be the orbifold complex structures of $N_{\nu}^{\prime}$ and $N_{\nu}$, respectively.

Proposition 2.3. $\psi$ is an isomorphism of complex orbifolds $\left(N_{\nu}^{\prime}, J^{N_{\nu}^{\prime}}\right) \rightarrow$ $\left(N_{\nu}, J^{N_{\nu}}\right)$.

Proof of Proposition 2.3. By Corollary 2.9, any $\tilde{T}$-orbit in $\tilde{A}_{\nu}^{\vee}$ intersects $X_{\boldsymbol{\nu}}$; thus $\psi$ is surjective.

To prove that $\psi$ is injective, suppose by contradiction that there exist $x_{1}, x_{2} \in X_{\nu}$ such that $x_{2} \in \tilde{T} \cdot x_{1}$ (i.e., $\psi\left(T \cdot x_{1}\right)=\psi\left(T \cdot x_{2}\right)$ ), but $x_{2} \notin T \cdot x_{1}$ (i.e., $T \cdot x_{1} \neq T \cdot x_{2}$ ). Perhaps after replacing $x_{2}$ with another point in $T \cdot x_{2}$, we may assume that $x_{2}=\tilde{\mu}_{e^{-\boldsymbol{\xi}}}^{A_{0}^{\vee}}\left(x_{1}\right)$ for some $\boldsymbol{\xi} \in \mathbb{R}^{r} \backslash\{\mathbf{0}\}$. We may write uniquely $\boldsymbol{\xi}=\boldsymbol{\xi}^{\prime}+a \boldsymbol{\nu}$, where $\boldsymbol{\xi}^{\prime} \in \boldsymbol{\nu}^{\perp}$ and $a \in \mathbb{R}$. Perhaps interchanging $x_{1}$ and $x_{2}$, we may assume without loss that $a \geq 0$.

Let us set $\boldsymbol{\eta}:=\imath \boldsymbol{\xi} \in \mathfrak{t}$. Considering the associated vector fields $\boldsymbol{\xi}_{A^{\vee}}, \boldsymbol{\eta}_{A^{\vee}} \in$ $\mathfrak{X}\left(A^{\vee}\right)$ we have $-\boldsymbol{\xi}_{A^{\vee}}=J^{\prime}\left(\boldsymbol{\eta}_{A^{\vee}}\right)$; hence $-\boldsymbol{\xi}_{A^{\vee}}$ is the gradient vector field of the Hamiltonian function $\tilde{\Phi}^{\boldsymbol{\eta}}=\langle\tilde{\Phi}, \boldsymbol{\eta}\rangle$, where $\tilde{\Phi}$ is as in (20).

Since $x_{1} \in X_{\nu}$, we have $\tilde{\Phi}\left(x_{1}\right)=\imath \lambda \nu$ for some $\lambda>0$, hence $\tilde{\Phi}^{\eta}\left(x_{1}\right)=$ $\lambda a\|\boldsymbol{\nu}\|^{2} \geq 0$. Since $\tilde{\Phi}^{\eta}$ is strictly increasing along its gradient flow where the gradient is non-vanishing,

$$
\tilde{\Phi}^{\eta}\left(\tilde{\mu}_{e^{-t} \boldsymbol{\xi}}^{A_{\boldsymbol{\xi}}^{\vee}}\left(x_{1}\right)\right)>\tilde{\Phi}^{\eta}\left(x_{1}\right) \geq 0 \quad \forall t>0 .
$$

On the other hand, we have

$$
\boldsymbol{\eta}_{A_{0}^{\vee}}=\boldsymbol{\eta}_{M}^{\sharp}-\tilde{\Phi}^{\eta} \partial_{\theta} \quad \Rightarrow \quad-\boldsymbol{\xi}_{A^{\vee}}=\left(J \boldsymbol{\eta}_{M}\right)^{\sharp}+\tilde{\Phi}^{\boldsymbol{\eta}} r \partial_{r} .
$$

Here $r \partial_{r}$ is the generator of the 1-parameter group of diffeomorphisms $\ell \mapsto$ $e^{t} \ell$. With $\varrho$ as in (16), for every $t>0$ we have

$$
-\boldsymbol{\xi}_{A^{\vee}}(\varrho)\left(\tilde{\mu}_{e^{-t} \boldsymbol{\xi}}^{A^{\vee}}\left(x_{1}\right)\right)=\tilde{\Phi}^{\eta}\left(\tilde{\mu}_{e^{-t \boldsymbol{\xi}}}^{A_{0}^{\vee}}\left(x_{1}\right)\right) r \partial_{r} \varrho\left(\tilde{\mu}_{e^{-t \boldsymbol{\xi}}}^{A^{\vee}}\left(x_{1}\right)\right)>0 .
$$

It follows that $\varrho\left(\tilde{\mu}_{e^{-t \xi}}^{A_{0}^{\vee}}\left(x_{1}\right)\right)>\varrho\left(x_{1}\right)=1$ for $t>0$; taking $t=1$, we conclude that $x_{2} \notin X$, a contradiction. Hence $\psi$ is a bijection. 
Let us verify that $\psi$ is a homeomorphism. The open sets of $N_{\nu}^{\prime}$ have the form $U / T$, where $U \subseteq X_{\nu}$ is open and $T$-invariant, and the open sets of $N_{\nu}$ have the form $\tilde{U} / \tilde{\tilde{T}}$, where $\tilde{U} \subseteq A_{\nu}^{\vee}$ is open and $\tilde{T}$-invariant. The previous argument shows that each $\tilde{T}$-orbit in $\tilde{A}_{\nu}^{\vee}$ intersects $X_{\nu}$ in a single $T$-orbit. One can see from this (and the definition of $\tilde{A}_{\nu}^{\vee}$ ) that there is a bijection between the family of $\tilde{T}$-invariant open sets $\tilde{U}$ in $\tilde{A}_{\nu}^{\vee}$ and the family of $T$-invariant open sets $U$ in $X_{\nu}$ given by $\tilde{U} \mapsto U:=\tilde{U} \cap X_{\nu}$, with inverse $U \mapsto \tilde{U}:=\tilde{T} \cdot U$.

Given any such $\tilde{U}$, we have $\psi^{-1}(\tilde{U} / \tilde{T})=U / T \subseteq N_{\nu}^{\prime}$, implying that $\psi$ is continuous. Similarly, given any such $U$ we have $\psi(U / T)=\tilde{U} / \tilde{T}$, implying that $\psi$ is open. Hence $\psi$ is a homeomorphism.

To conclude that $\psi$ is an isomorphism of complex orbifolds, it suffices to verify that its local expressions in uniformizing charts are biholomorphisms; actually, it suffices to do so for corresponding defining families in the sense of [S1] and [S2] that cover $N_{\nu}^{\prime}$ and $N_{\nu}$. Let $F$ be a slice at $x$ for $\mu^{X_{\nu}}$ at some $x \in X_{\boldsymbol{\nu}}$; by Proposition 2.2. perhaps after shrinking $F$ if necessary, we may assume that $F$ is also a slice at $x$ for $\tilde{\mu}^{\vee}$. Hence $\left(F, J^{F}\right)$ is a uniformizing chart of both $N_{\nu}^{\prime}$ and $N_{\nu}$. By definition of $\psi$ and the previous considerations, the identity $\operatorname{id}_{F}: F \rightarrow F$ is a local representative map of $\psi$, and it is obviously biholomorphic $\left(F, J^{F}\right) \rightarrow\left(F, J^{F}\right)$.

The sheaf of holomorphic functions on $N_{\nu}$ is defined equivalently by the $\tilde{T}$-invariant holomorphic functions on $\tilde{A}_{\nu}^{\vee}$ or the $T_{\ell}$-invariant holomorphic functions on the slices $\left(F, J^{F}\right)$. Let us briefly clarify this point.

Since $\left(F, J^{F}\right)$ is generally not a complex submanifold of $\left(A^{\vee}, J^{\prime}\right)$, arbitrary holomorphic functions on the saturation $\tilde{T} \cdot F$ needn't restrict to holomorphic functions on $\left(F, J^{F}\right)$. However, this does happens if we restrict to invariant holomorphic functions.

Definition 2.2. Suppose that $\ell \in A_{\nu}^{\vee}$ and that $F \subseteq A_{\nu}^{\vee}$ is a slice at $\ell$ for $\tilde{\mu}^{\tilde{A}_{\nu}}$. Let us adopt the following notation.

1. $\mathcal{O}(F)$ is the ring of $J^{F}$-holomorphic functions on $F$;

2. $\mathcal{O}(F)^{T_{\ell}} \subseteq \mathcal{O}(F)$ is the subring of $T_{\ell}$-invariant functions in $\mathcal{O}(F)$;

3. $\mathcal{O}(\tilde{T} \cdot F)$ is the ring of $J^{\prime}$-holomorphic functions on the saturation of $F$ under $\tilde{\mu}^{A^{\vee}}$;

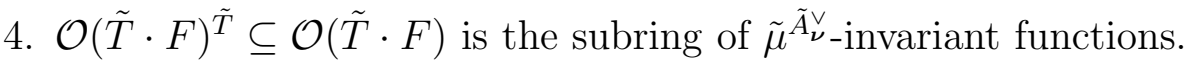

Then we have the following, whose prooof will be omitted (see the argument for Proposition 2.4). 
Lemma 2.7. In the situation of Definition 2.2, restriction yields an isomorphism $\mathcal{O}(\tilde{T} \cdot F)^{\tilde{T}} \rightarrow \mathcal{O}(F)^{T_{\ell}}$.

\subsection{Holomorphic and CR functions on $\tilde{A}_{\nu}^{\vee}$ and $X_{\nu}$}

$M_{\nu}$ is a CR submanifold of $M$, and the maximal complex sub-bundle $\mathcal{H}\left(M_{\nu}\right) \subseteq$ $T M_{\boldsymbol{\nu}}$ has complex dimension $d+1-r$, and is as follows. If $m \in M_{\boldsymbol{\nu}}$, $\left(\tilde{t}_{\boldsymbol{\nu}^{\perp}}^{r-1}\right)_{M}(m) \subseteq T_{m} M_{\boldsymbol{\nu}}$ is a complex subspace of dimension $r-1$, since $\tilde{\gamma}^{M}$ is locally free at $m$. Then

$$
\mathcal{H}\left(M_{\boldsymbol{\nu}}\right)_{m}=\left(\tilde{t}_{\boldsymbol{\nu}^{\perp}}^{r-1}\right)_{M}(m)^{\perp_{h_{m}}}
$$

where $h_{m}=g_{m}-\imath \omega_{m}$ is the Hermitian product on $T_{m} M$ associated to the Kähler metric.

Similarly, $X_{\nu}$ is a CR submanifold of $A^{\vee}$. The maximal complex subbundle $\mathcal{H}\left(X_{\nu}\right) \subset T X_{\nu}$ is as follows. If $x \in X_{\nu}$ and $m=\pi(x)$, then

$$
\mathcal{H}\left(X_{\nu}\right)_{x}=\mathcal{H}\left(M_{\nu}\right)_{m}^{\sharp}
$$

Definition 2.3. Let be given $\boldsymbol{\lambda} \in \mathbb{Z}^{r}$.

For any $\tilde{T}$-invariant open subset $\tilde{U} \subseteq \tilde{A}_{\boldsymbol{\nu}}^{\vee}$, let $\mathcal{O}(\tilde{U})_{\boldsymbol{\lambda}}$ be the ring of holomorphic functions $\tilde{S}: \tilde{U} \rightarrow \mathbb{C}$ such that

$$
\tilde{S}\left(\tilde{\mu}_{\tilde{\mathbf{t}}^{-1}}^{A^{\vee}}(\ell)\right)=\tilde{\chi}_{\boldsymbol{\lambda}}(\tilde{\mathbf{t}}) \tilde{S}(\ell) \quad(\tilde{\mathbf{t}} \in \tilde{T}, \ell \in \tilde{U}) .
$$

For any $T$-invariant open subset $U \subseteq X_{\boldsymbol{\nu}}$, let let $\mathcal{C R}(U)_{\boldsymbol{\lambda}}$ be the ring of CR functions on $U$ satisfying

$$
S\left(\mu_{\mathbf{t}^{-1}}^{c}(x)\right)=\chi_{\boldsymbol{\lambda}}(\mathbf{t}) S(x) \quad\left(\mathbf{t} \in T, x \in X_{\boldsymbol{\nu}}\right) .
$$

Proposition 2.4. With notation in Definition 2.3, suppose that $U=\tilde{U} \cap X_{\boldsymbol{\nu}}$. Then restriction yields a ring isomorphism $\mathcal{O}(\tilde{U})_{\boldsymbol{\lambda}} \rightarrow \mathcal{C} \mathcal{R}(U)_{\boldsymbol{\lambda}}$.

Corollary 2.15. Restriction yields an isomorphism $\mathcal{O}\left(\tilde{A}_{\boldsymbol{\nu}}^{\vee}\right)_{\boldsymbol{\lambda}} \rightarrow \mathcal{C R}\left(X_{\boldsymbol{\nu}}\right)_{\boldsymbol{\lambda}}$.

Proof of Proposition 2.4. Clearly if $\tilde{S} \in \mathcal{O}(\tilde{U})_{\boldsymbol{\lambda}}$ then $S:=\left.\tilde{S}\right|_{U} \in \mathcal{C} \mathcal{R}(U)_{\boldsymbol{\lambda}}$. Thus the ring homomorphim in the statement is well-defined and obviously injective.

To prove surjectivity, suppose conversely that $S \in \mathcal{C} \mathcal{R}(U)_{\lambda}$. Let us define $\tilde{S}: \tilde{U}=\tilde{T} \cdot U \rightarrow \mathbb{C}$ by setting

$$
\tilde{S}\left(\tilde{\mu}_{\tilde{\mathbf{t}}}^{A_{0}^{\vee}}(x)\right)=\tilde{\chi}_{-\boldsymbol{\lambda}}(\tilde{\mathbf{t}}) S(x)=\tilde{\chi}_{\boldsymbol{\lambda}}(\tilde{\mathbf{t}})^{-1} S(x) \quad\left(\tilde{\mathbf{t}} \in \tilde{T}, \quad x \in X_{\boldsymbol{\nu}}\right) .
$$


To verify that (28) is well-defined, suppose that $\tilde{\mu}_{\tilde{\mathbf{t}}_{1}}^{A_{0}^{\vee}}\left(x_{1}\right)=\tilde{\mu}_{\tilde{\mathbf{t}}_{2}}^{A_{0}^{\vee}}\left(x_{2}\right)$ with $\tilde{\mathbf{t}}_{j} \in \tilde{T}$ and $x_{j} \in U$. By the argument in the proof of Proposition 2.3, $\tilde{\mathbf{t}}_{2}^{-1} \tilde{\mathbf{t}}_{1} \in T$. Therefore

$$
S\left(x_{2}\right)=\chi_{-\boldsymbol{\lambda}}\left(\tilde{\mathbf{t}}_{2}^{-1} \tilde{\mathbf{t}}_{1}\right) S\left(x_{1}\right)=\tilde{\chi}_{-\boldsymbol{\lambda}}\left(\tilde{\mathbf{t}}_{2}\right)^{-1} \tilde{\chi}_{-\boldsymbol{\lambda}}\left(\tilde{\mathbf{t}}_{1}\right) S\left(x_{1}\right) .
$$

By construction, $\tilde{S}$ satisfies (26) and restricts to $S$ on $U$. To prove that $S \mapsto \tilde{S}$ inverts restriction it remains to verify that $\tilde{S}$ is holomorphic, i.e. that $\mathrm{d}_{\ell} \tilde{S}$ is $\mathbb{C}$-linear for any $\ell \in \tilde{U}$.

By Corollary 2.9, the map

$$
\mathcal{F}:(\tilde{\mathbf{t}}, x) \in \tilde{T} \times X_{\nu} \mapsto \tilde{\mu}_{\tilde{\mathbf{t}}}^{A_{0}^{\vee}}(x) \in \tilde{A}_{\nu}^{\vee}
$$

is surjective. In fact, $\mathcal{F}$ exhibits $\tilde{A}_{\nu}^{\vee}$ as the quotient of $\tilde{T} \times X_{\nu}$ by the free action of $T$ given by

$$
\mathbf{t} \cdot(\tilde{\mathbf{t}}, x):=\left(\tilde{\mathbf{t}} \mathbf{t}^{-1}, \mu_{\mathbf{t}}^{X}(x)\right) .
$$

Furthermore, $\mathcal{F}(\tilde{T} \times U)=\tilde{U}$.

For every $\tilde{\mathbf{t}} \in \tilde{T}$ let us set

$$
X_{\nu}^{\tilde{\mathbf{t}}}:=\mathcal{F}\left(\{\tilde{\mathbf{t}}\} \times X_{\nu}\right)=\tilde{\mu}_{\tilde{\mathbf{t}}}^{A^{\vee}}\left(X_{\nu}\right) .
$$

Again by the proof of Proposition 2.3 we have $X_{\nu}^{\tilde{\mathbf{t}}_{1}}=X_{\boldsymbol{\nu}}^{\tilde{\mathbf{t}}_{2}}$ if $\tilde{\mathbf{t}}_{1}^{-1} \tilde{\mathbf{t}}_{2} \in T$, and $X_{\nu}^{\tilde{\mathbf{t}}_{1}} \cap X_{\nu}^{\tilde{\mathbf{t}}_{2}}=\emptyset$ otherwise. Clearly $X_{\nu}^{\tilde{\mathbf{t}}}$ is a CR submanifold of $A^{\vee}$, and its CR bundle $\mathcal{H}\left(X_{\boldsymbol{\nu}}^{\tilde{\mathrm{t}}}\right)$ is as follows. If $\ell=\mathcal{F}(\tilde{\mathbf{t}}, x) \in X_{\boldsymbol{\nu}}^{\tilde{\mathbf{t}}}$, then

$$
\mathcal{H}\left(X_{\nu}^{\tilde{\mathbf{t}}}\right)_{\ell}=\mathrm{d}_{x} \tilde{\mu}_{\tilde{\mathbf{t}}}^{A^{\vee}}\left(\mathcal{H}\left(X_{\nu}\right)_{x}\right) .
$$

If $\tilde{\mathbf{t}} \in \tilde{T}$, let us identify $T_{\tilde{\mathbf{t}}} \tilde{T} \cong \tilde{\mathfrak{t}}$ in the standard manner. For $(\tilde{\mathbf{t}}, x) \in$ $\tilde{T} \times X_{\nu}$, let us consider the vector subspace

$$
\mathcal{K}(\tilde{\mathbf{t}}, x):=\tilde{\mathfrak{t}} \times \mathcal{H}\left(X_{\boldsymbol{\nu}}\right)_{x} \subseteq T_{\tilde{\mathbf{t}}} \tilde{T} \times T_{x} X_{\boldsymbol{\nu}} \cong T_{(\tilde{\mathbf{t}}, x)}\left(\tilde{T} \times X_{\boldsymbol{\nu}}\right) .
$$

The distribution $\mathcal{K} \subseteq T\left(\tilde{T} \times X_{\boldsymbol{\nu}}\right)$ is invariant under (29) and is naturally a complex vector bundle; furthermore, $\mathrm{d} \mathcal{F}$ yields an isomorphism of complex vector bundles $\mathcal{K} \rightarrow \mathcal{F}^{*}\left(T \tilde{A}_{\nu}^{\vee}\right)$. More explicitly, if $\ell=\mathcal{F}(\tilde{\mathbf{t}}, x)$ then

$$
\left.\mathrm{d}_{(\tilde{\mathbf{t}}, x)} \mathcal{F}\right|_{\mathcal{K}(\tilde{t}, x)}: \tilde{\mathfrak{t}} \times \mathcal{H}\left(X_{\nu}\right)_{x} \rightarrow \tilde{\mathfrak{t}}_{A^{\vee}}(\ell) \oplus \mathcal{H}\left(X_{\nu}^{\tilde{\mathfrak{t}}}\right)_{\ell}=T_{\ell} A^{\vee}
$$

is an isomorphism of complex vector spaces, respecting the direct sum decompositions on both sides. 
Given $S \in \mathcal{C R}(U)_{\boldsymbol{\lambda}}$, let us consider the complex function $\hat{S}$ on $\tilde{T} \times X_{\boldsymbol{\nu}}$ given by

$$
\hat{S}(\tilde{\mathbf{t}}, x):=\tilde{\chi}_{\nu}(\tilde{\mathbf{t}})^{-1} S(x) .
$$

Then $\hat{S}=\tilde{S} \circ \mathcal{F}$.

Let us assume that $\ell=\mathcal{F}(\tilde{\mathbf{t}}, x)$. We have

$$
\left.\mathrm{d}_{(\tilde{\mathbf{t}}, x)} \hat{S}\right|_{\mathcal{H}(\tilde{\mathbf{t}}, x)}=\left.\mathrm{d}_{\ell} \tilde{S} \circ \mathrm{d}_{(\tilde{\mathbf{t}}, x)} \mathcal{F}\right|_{\mathcal{K}(\tilde{\mathbf{t}}, x)}: \mathcal{K}(\tilde{\mathbf{t}}, x) \rightarrow \mathbb{C}
$$

Hence to prove that $\mathrm{d}_{\ell} \tilde{S}: T_{\ell} A_{\nu}^{\vee} \rightarrow \mathbb{C}$ is $\mathbb{C}$-linear it suffices to show that $\mathrm{d}_{(\tilde{\mathbf{t}}, x)} \hat{S}$ is $\mathbb{C}$-linear on $\mathcal{K}_{(\tilde{\mathbf{t}}, x)}=\tilde{\mathfrak{t}} \times \mathcal{H}\left(X_{\nu}\right)_{x}$; to do so, in turn it is sufficient to verify $\mathbb{C}$-linearity on each summand $\tilde{\mathfrak{t}}$ and $\mathcal{H}\left(X_{\boldsymbol{\nu}}\right)_{x}$ separately. This follows from (31), since $\tilde{\chi}_{\nu}$ is holomorphic (implying $\mathbb{C}$-linearity on the first summand) and $S$ is CR (implying $\mathbb{C}$-linearity on the second summand).

\subsection{The orbifold line bundles $B_{\nu}$ and $B_{\nu}^{\prime}$}

We have seen that the restrictions of $\mu^{X}$ to $X_{\nu}$ and of $\tilde{\mu}^{A^{\vee}}$ to $\tilde{A}_{\nu}^{\vee}$ are locally free, effective and proper actions of $T$ and $\tilde{T}$, respectively, and that the corresponding quotients $N_{\nu}^{\prime}:=X_{\nu} / T$ and $N_{\nu}:=\tilde{A}_{\nu}^{\vee} / \tilde{T}$ are naturally isomorphic complex orbifolds. Furthermore, the projections $p_{\nu}^{\prime}: X_{\nu} \rightarrow N_{\nu}^{\prime}$ and $p_{\boldsymbol{\nu}}: \tilde{A}_{\nu}^{\nu} \rightarrow N_{\nu}$ are principal $V$-bundles with structure group $T$ and $\tilde{T}$, respectively.

Associated to the characters $\chi_{\nu}: T \rightarrow S^{1}$ and $\tilde{\chi}_{\nu}: \tilde{T} \rightarrow \mathbb{C}^{*}$, we have 1dimensional representations of $T$ and $\tilde{T}$, respectively; we shall denote either one by $\mathbb{C}_{\nu}$. The product actions $\mu^{X \times \mathbb{C}_{\nu}}$ and $\tilde{\mu}^{A^{\vee} \times \mathbb{C}_{\nu}}$ are therefore also locally free, effective and proper on $X_{\nu} \times \mathbb{C}_{\nu}$ and $\tilde{A}_{\nu}^{\vee} \times \mathbb{C}_{\nu}$ respectively. Hence the quotients $B_{\nu}^{\prime}:=X_{\nu} \times_{T} \mathbb{C}_{\boldsymbol{\nu}}$ and $B_{\boldsymbol{\nu}}:=\tilde{A}_{\boldsymbol{\nu}}^{\vee} \times_{\tilde{T}} \mathbb{C}_{\boldsymbol{\nu}}$ are orbifold line bundles on $N_{\nu}^{\prime}$ and $N_{\nu}$. Let us denote by

$$
P_{\nu}^{\prime}: B_{\nu}^{\prime} \rightarrow N_{\nu}^{\prime} \quad \text { and } \quad P_{\nu}: B_{\nu} \rightarrow N_{\nu}
$$

the respective projections.

Lemma 2.8. Suppose $x \in X_{\nu}$ and let $F \subseteq X_{\nu}$ be a slice for the restriction of $\mu^{X}$ to $X_{\nu}$. Then $F \times \mathbb{C}_{\nu}$ is a slice at $(x, 0)$ for the restriction of $\mu^{X \times \mathbb{C}_{\nu}}$ to $X_{\boldsymbol{\nu}} \times \mathbb{C}_{\boldsymbol{\nu}}$. The collection of all these slices yields a defining family for $B_{\boldsymbol{\nu}}^{\prime}$.

Similarly, suppose $\ell \in \tilde{A}_{\nu}^{\vee}$ and let $F \subseteq \tilde{A}_{\nu}^{\vee}$ be a slice at $\ell$ for the restriction of $\tilde{\mu}^{A^{\vee}}$ to $\tilde{A}_{\nu}^{\vee}$. Then $F \times \mathbb{C}_{\nu}$ is a slice at $(\ell, 0)$ for the restriction of $\tilde{\mu}^{A^{\vee} \times \mathbb{C}_{\nu}}$ to $\tilde{A}_{\boldsymbol{\nu}}^{\vee} \times \mathbb{C}_{\boldsymbol{\nu}}$. The collection of all these slices yields a defining family for $B_{\boldsymbol{\nu}}$. 
Proof of Lemma 2.8. Let us consider the former statement, the proof of the latter being similar.

Since $F \times \mathbb{C}_{\nu}$ is transverse to the $T$-orbits in $X_{\boldsymbol{\nu}} \times \mathbb{C}_{\boldsymbol{\nu}}$, the map $h: T \times$ $\left(F \times \mathbb{C}_{\nu}\right) \rightarrow X_{\nu} \times \mathbb{C}_{\nu}$ induced by the diagonal action is a local diffeomorphism onto the open saturation $T \cdot\left(F \times \mathbb{C}_{\nu}\right) \subseteq X_{\boldsymbol{\nu}} \times \mathbb{C}$.

Clearly we have the equality of stabilizers $T_{(x, 0)}=T_{x}$. Furthermore, suppose $(y, w) \in F \times \mathbb{C}_{\boldsymbol{\nu}}, \mathbf{t} \in T$. Then

$$
\mu_{\mathbf{t}}^{X \times \mathbb{C}_{\nu}}(y, w)=\left(\mu_{\mathbf{t}}^{X}(y), \chi_{\nu}(\mathbf{t}) w\right) \in F \times \mathbb{C}_{\nu}
$$

if and only if $\mu_{\mathbf{t}}^{X}(y) \in F$, that is, if and only if $\mathbf{t} \in T_{x}$. Hence $h$ descends to a diffeomorphism

$$
\bar{h}:\left(T \times\left(F \times \mathbb{C}_{\boldsymbol{\nu}}\right)\right) / T_{x} \rightarrow T \cdot\left(F \times \mathbb{C}_{\boldsymbol{\nu}}\right),
$$

where $T_{x}$ acts antidiagonally on $T \times\left(F \times \mathbb{C}_{\boldsymbol{\nu}}\right)$.

Corollary 2.16. $F \times \mathbb{C}_{\nu}$ with the diagonal action of $T_{x}$ uniformizes the open set $\left(F \times \mathbb{C}_{\boldsymbol{\nu}}\right) / T_{x} \subseteq B_{\boldsymbol{\nu}^{\prime}}$. The collection of all these uniformizing charts is a defnining family for the orbifold line bundle $B_{\boldsymbol{\nu}}^{\prime}$. A similar statement holds fo $B_{\boldsymbol{\nu}}$.

Given the complex structure $J^{F}$ on each $F$ (Lemma 2.5), we obtain a product complex structure on $F \times \mathbb{C}_{\nu}$. Hence both $B_{\boldsymbol{\nu}}^{\prime}$ and $B_{\boldsymbol{\nu}}$ are complex orbifolds of complex dimension $d+2-r$.

Since any $T$-orbit in $X_{\nu} \times \mathbb{C}$ is contained in a unique $\tilde{T}$-orbit in $\tilde{A}_{\nu}^{\vee} \times \mathbb{C}$, there is a natural continuous map $\tilde{\psi}: B_{\nu}^{\prime} \rightarrow B_{\nu}$. The proof of Proposition 2.3 can be adapted to yield the following:

Proposition 2.5. $\tilde{\psi}$ is an isomorphism of complex orbifolds, and $\psi \circ P_{\nu}^{\prime}=$ $P_{\nu} \circ \tilde{\psi}$.

\subsection{The orbifold circle bundle $Y_{\nu}$}

We need an alternative description of $B_{\nu}^{\prime}$. Consider the intermediate quotient

$$
Y_{\nu}:=X_{\nu} / T_{\nu^{\perp}}^{r-1}
$$

then $Y_{\nu}$ is compact orbifold, of (real) dimension $2(d+1-r)+1$, and the integrable and invariant $\mathrm{CR}$ structure on $X_{\nu}$ descends to an integrable CR structure on $Y_{\boldsymbol{\nu}}$. We shall denote by $\mathcal{H}\left(Y_{\boldsymbol{\nu}}\right)$ the CR bundle of $Y_{\boldsymbol{\nu}}$. 
Let $T_{\nu}^{1} \leqslant T$ be the connected compact subgroup of $T$ associated to the Lie subalgebra $\operatorname{span}(\imath \boldsymbol{\nu}) \subseteq \mathfrak{t}$. Given that $\boldsymbol{\nu}$ is coprime, we have a Lie group isomorphism

$$
\kappa_{\boldsymbol{\nu}}: e^{\imath \vartheta} \in S^{1} \mapsto e^{\imath \vartheta \nu}:=\left(e^{\imath \vartheta \nu_{1}}, \ldots, e^{\imath \vartheta \nu_{r}}\right) \in T_{\boldsymbol{\nu}}^{1} .
$$

Let us set $\bar{T}_{\nu}^{1}:=T / T_{\nu^{\perp}}^{r-1} \cong T_{\nu}^{1} /\left(T_{\nu}^{1} \cap T_{\nu^{\perp}}^{r-1}\right)$.

Suppose $x \in X_{\nu}$, and let $F \subseteq X_{\nu}$ be a slice at $x$ for the restriction of $\gamma^{X}$ to $X_{\nu}$. We can view $T \times F$ as a uniformizing chart for the smooth orbifold $X_{\nu}$, with uniformized open set $T \cdot F=(T \times F) / T_{x}$. Then $\bar{T}_{\nu}^{1} \times F$ is a uniformizing chart for $Y_{\boldsymbol{\nu}}$, covering the open set $(T \cdot F) / T_{\boldsymbol{\nu}^{\perp}}^{r-1}$.

Explicitly, $T_{x}$ act effectively on $\bar{T}_{\nu}^{1} \times F$ by

$$
t_{0} \cdot(\bar{t}, f):=\left(\bar{t} \bar{t}_{0}^{-1}, \mu_{t_{0}}^{X}(f)\right)
$$

where for any $t \in T$ we have set $\bar{t}=t T_{\boldsymbol{\nu}^{\perp}}^{r-1} \in \bar{T}_{\boldsymbol{\nu}}^{1}$. Then the map

$$
\gamma:(\bar{t}, f) \in \bar{T}_{\nu}^{1} \times F \mapsto T_{\nu^{\perp}}^{r-1} \cdot \mu_{t}^{X}(f) \in(T \cdot F) / T_{\nu^{\perp}}^{r-1} \subseteq Y_{\nu}
$$

induces a homeomorphism $(T \cdot F) / T_{\nu^{\perp}}^{r-1}=\left(\bar{T}^{1} \times F\right) / T_{\ell}$. Letting $F$ vary, we obtain a defining family for $Y_{\nu}$.

Furthermore, $\bar{T}_{\nu}^{1}$ acts effectively on $Y_{\boldsymbol{\nu}}$, and $N_{\nu}^{\prime}=Y_{\boldsymbol{\nu}} / \bar{T}_{\nu}^{1}$; let $\sigma_{\boldsymbol{\nu}}: Y_{\boldsymbol{\nu}} \rightarrow$ $N_{\nu}^{\prime}$ be the projection. For each slice $F \subseteq X_{\nu}$, as above, the local representation of $\sigma_{\nu}$ is the projection $\bar{T}_{\nu}^{1} \times F \rightarrow F$. Thus $Y_{\nu}$ is a principal $V$-bundle over $N_{\nu}^{\prime}$, with structure group $\bar{T}_{\nu}^{1}$.

Being trivial on $T_{\nu^{\perp}}^{r-1}, \chi_{\nu}$ descends to a character $\chi_{\nu}^{\prime}: \bar{T}_{\nu}^{1} \rightarrow S^{1}$.

Lemma 2.9. Given that $\boldsymbol{\nu}$ is coprime, $\chi_{\boldsymbol{\nu}}^{\prime}$ is a Lie group isomorphism.

Proof of Lemma 2.9. Since $\bar{T}_{\boldsymbol{\nu}}^{1} \cong T_{\boldsymbol{\nu}}^{1} /\left(T_{\boldsymbol{\nu}}^{1} \cap T_{\boldsymbol{\nu}^{\perp}}^{r-1}\right)$, the statement is equivalent to the equality

$$
\operatorname{ker}\left(\left.\chi_{\nu}\right|_{T_{\nu}^{1}}\right)=T_{\nu}^{1} \cap T_{\nu^{\perp}}^{r-1}
$$

since clearly $T_{\boldsymbol{\nu}^{\perp}}^{r-1} \subseteq \operatorname{ker}\left(\chi_{\boldsymbol{\nu}}\right)$, we need only prove that $\operatorname{ker}\left(\left.\chi_{\boldsymbol{\nu}}\right|_{T_{\nu}^{1}}\right) \subseteq T_{\boldsymbol{\nu}^{1}}^{1} \cap T_{\boldsymbol{\nu}^{\perp}}^{r-1}$. Since $\boldsymbol{\nu}$ is coprime, there exists $\mathbf{k}=\left(\begin{array}{lll}k_{1} & \cdots & k_{r}\end{array}\right) \in \mathbb{Z}^{r}$ such that $\langle\boldsymbol{\nu}, \mathbf{k}\rangle=$ $\sum_{j=1}^{r} k_{j} \nu_{j}=1$.

Let $\kappa_{\nu}$ be as in (33). Then

$$
\chi_{\boldsymbol{\nu}} \circ \kappa_{\boldsymbol{\nu}}\left(e^{\imath \vartheta}\right)=\chi_{\boldsymbol{\nu}}\left(e^{\imath \vartheta \boldsymbol{\nu}}\right)=e^{\imath \vartheta\|\boldsymbol{\nu}\|^{2}} \quad\left(e^{\imath \vartheta} \in S^{1}\right) .
$$


Hence if $e^{\imath \vartheta \boldsymbol{\nu}} \in \operatorname{ker}\left(\chi_{\boldsymbol{\nu}}\right)$, then we may assume $\vartheta=\vartheta_{j}:=2 \pi j /\|\boldsymbol{\nu}\|^{2}$ for some $j=0, \ldots,\|\boldsymbol{\nu}\|^{2}-1$. We have

$$
\left\langle\vartheta_{j} \boldsymbol{\nu}, \boldsymbol{\nu}\right\rangle=\frac{2 \pi j}{\|\boldsymbol{\nu}\|^{2}}\langle\boldsymbol{\nu}, \boldsymbol{\nu}\rangle=2 \pi j=2 \pi j\langle\mathbf{k}, \boldsymbol{\nu}\rangle,
$$

so that $\vartheta_{j} \boldsymbol{\nu}-2 \pi j \mathbf{k} \in \boldsymbol{\nu}^{\perp}$. Thus

$$
e^{\imath \vartheta_{j} \boldsymbol{\nu}}=e^{\imath\left[\vartheta_{j} \boldsymbol{\nu}-2 \pi j \mathbf{k}\right]} \in T_{\boldsymbol{\nu}}^{1} \cap T_{\boldsymbol{\nu}^{\perp}}^{r-1} .
$$

Since $k_{\nu}$ is an isomorphism, (35) implies the following.

Corollary 2.17. Assuming that $\boldsymbol{\nu}$ is coprime,

$$
\left|T_{\nu^{\perp}}^{r-1} \cap T_{\nu}^{1}\right|=\|\nu\|^{2} .
$$

Given the isomorphism $\chi_{\nu}^{\prime}: \bar{T}_{\nu}^{1} \cong S^{1}$, we shall view $Y_{\nu}$ as a principal $V$-bundle over $N_{\nu}^{\prime}$ with structure group $S^{1}$. Let us denote by

$$
\sigma^{Y_{\nu}}: S^{1} \times Y_{\nu} \rightarrow Y_{\nu}
$$

the corresponding action.

Let

$$
Q_{\nu}: X_{\nu} \rightarrow Y_{\nu}
$$

be the projection. Then $U$ is a $T$-invariant open subset of $X_{\nu}$ if and only if its image $Q_{\boldsymbol{\nu}}(U)$ is a $\bar{T}_{\boldsymbol{\nu}}^{1} \cong S^{1}$-invariant open subset of $Y_{\boldsymbol{\nu}}$. It follows (recall the proof of Proposition 2.3) that there is a bijective correspondence between $\tilde{T}$-invariant open subsets $\tilde{U} \subseteq \tilde{A}_{\nu}^{\vee}, T$-invariant open subsets $U \subseteq X_{\nu}$, $S^{1}$-invariant open subsets $\bar{U} \subseteq Y_{\nu}$, given by $U ;=\tilde{U} \cap X_{\nu}, \bar{U}:=Q_{\nu}(U)$.

The character $\chi_{k \nu}^{\prime}=\left(\chi_{\nu}^{\prime}\right)^{k}: \bar{T}_{\nu}^{1} \rightarrow S^{1}$ corresponds to the endomorphism $\chi_{k}: g \in S^{1} \mapsto g^{k} \in S^{1}$. Let us denote by $\mathcal{C R}\left(Y_{\nu}\right)$ the collection of all CR functions on $Y_{\boldsymbol{\nu}}$, and for any $k \in \mathbb{Z}$ let us set

$$
\mathcal{C R}\left(Y_{\boldsymbol{\nu}}\right)_{k}=\left\{f \in \mathcal{C} \mathcal{R}\left(Y_{\boldsymbol{\nu}}\right): f \circ \sigma_{e^{-\imath \theta}}^{Y_{\boldsymbol{\nu}}}=e^{\imath k \theta} f, \quad \forall e^{\imath \theta} \in S^{1}\right\} .
$$

Using that the CR structure of $Y_{\nu}$ is obtained by descending the invariant CR structure of $X_{\nu}$, we can complement Proposition 2.4 and Corollary 2.15 by the following isomorphisms induced by pull-back:

$$
\mathcal{O}(\tilde{U})_{k \nu} \cong \mathcal{C R}(U)_{k \nu} \cong \mathcal{C R}(\bar{U})_{k} .
$$

Letting $H^{0}\left(N_{\boldsymbol{\nu}}, B_{k \boldsymbol{\nu}}\right)$ denote the space of holomorphic sections of the orbifold line bundle $B_{k \nu}$, we conclude that

$$
H^{0}\left(N_{\nu}, B_{k \nu}\right) \cong \mathcal{O}\left(\tilde{A}_{\nu}^{\vee}\right)_{k \nu} \cong \mathcal{C R}\left(X_{\nu}\right)_{k \nu} \cong \mathcal{C R}\left(Y_{\nu}\right)_{k}
$$




\subsection{The induced Kähler structure of $N_{\nu}$}

We shall see that $P_{\nu}: B_{\nu} \rightarrow N_{\nu}$ is a positive holomorphic $V$-line bundle. In view of Propositions 2.3 and 2.5 we may equivalently consider $P_{\nu}^{\prime}: B_{\nu}^{\prime} \rightarrow N_{\nu}^{\prime}$. With $\alpha$ as in (11), let $\alpha^{X_{\nu}}:=\jmath_{\nu}^{*}(\alpha)$, where

$$
\jmath_{\nu}: X_{\nu} \hookrightarrow X
$$

is the inclusion. Then $\alpha^{X_{\nu}}$ is $T$-invariant, and by definition of $X_{\nu}$ for any $\boldsymbol{\xi} \in \boldsymbol{\nu}^{\perp}$ we have

$$
\iota\left((\iota \boldsymbol{\xi})_{X_{\nu}}\right) \alpha^{X_{\nu}}=\jmath_{\nu}^{*}\left(\iota\left((\iota \boldsymbol{\xi})_{X}\right) \alpha\right)=-\langle\Phi, \imath \boldsymbol{\xi}\rangle \circ \jmath_{\nu}=0 .
$$

Hence $\alpha^{X_{\nu}}$ is the pull-back of an orbifold 1-form $\alpha^{Y_{\nu}}$ on $Y_{\boldsymbol{\nu}}$. Similarly, being $T$-invariant, $\Phi$ descends to a smooth function $\bar{\Phi}: Y_{\nu} \rightarrow \mathfrak{t}^{\vee}$; hence $\Phi^{\nu}=$ $\langle\Phi, \imath \boldsymbol{\nu}\rangle$ descends to a smooth function $\bar{\Phi}^{\nu}: Y_{\boldsymbol{\nu}} \rightarrow \mathbb{R}$.

Clearly,

$$
\iota\left((\iota \boldsymbol{\nu})_{Y_{\nu}}\right) \alpha^{Y_{\nu}}=-\bar{\Phi}^{\nu}
$$

Let us define

$$
\beta_{\boldsymbol{\nu}}:=\frac{\|\boldsymbol{\nu}\|^{2}}{\bar{\Phi}^{\nu}} \alpha^{Y_{\nu}}
$$

and let $-\delta^{Y_{\nu}} \in \mathfrak{X}\left(Y_{\boldsymbol{\nu}}\right)$ be the infintesimal generator of $\sigma^{Y_{\boldsymbol{\nu}}}$ in (36). Thus by Corollary 2.17

$$
-\delta^{Y_{\nu}}=\frac{1}{\|\boldsymbol{\nu}\|^{2}}(\imath \boldsymbol{\nu})_{Y_{\boldsymbol{\nu}}}
$$

Given (43) and (42), we conclude the following.

Corollary 2.18. $\beta_{\nu}$ is $\sigma^{Y_{\nu}}$-invariant, and $\beta_{\nu}\left(\delta^{Y_{\nu}}\right)=1$.

Hence $\beta_{\nu}$ is a connection 1 -form for the principal $V$-bundle $P_{\nu}^{\prime}: B_{\nu}^{\prime} \rightarrow N_{\nu}^{\prime}$. Explicitly,

$$
\mathrm{d} \beta_{\boldsymbol{\nu}}=\|\boldsymbol{\nu}\|^{2}\left[\frac{1}{\bar{\Phi}^{\nu}} \mathrm{d} \alpha^{Y_{\nu}}-\frac{1}{\left(\bar{\Phi}^{\nu}\right)^{2}} \mathrm{~d} \bar{\Phi}^{\nu} \wedge \alpha^{Y_{\nu}}\right]
$$

and one can also verify that $\iota\left(\delta^{Y_{\boldsymbol{\nu}}}\right) \mathrm{d} \beta_{\boldsymbol{\nu}}=0$ by direct inspection using (43) and (44). Furthermore, the kernel of $\beta_{\nu}$ is the CR bundle of $Y_{\nu}$ :

$$
\operatorname{ker}\left(\beta_{\boldsymbol{\nu}}\right)=\operatorname{ker}\left(\alpha^{Y_{\boldsymbol{\nu}}}\right)=\mathcal{H}\left(Y_{\boldsymbol{\nu}}\right)
$$

Hence we reach the following conclusion. Let $\pi_{\nu}: Y_{\nu} \rightarrow N_{\nu}^{\prime}$ be the projection.

Lemma 2.10. There exists a $(1,1)$-form $\eta_{\boldsymbol{\nu}}^{\prime}$ on $N_{\boldsymbol{\nu}}^{\prime}$ such that $\mathrm{d}\left(\beta_{\boldsymbol{\nu}}\right)=2 \pi_{\boldsymbol{\nu}}^{*}\left(\eta_{\boldsymbol{\nu}}^{\prime}\right)$. 
We shall denote by $\eta_{\nu}$ the corresponding form on $N_{\nu}$. With the notation of Proposition 2.3, we have the following.

Proposition 2.6. $\left(N_{\nu}^{\prime}, J^{N_{\nu}^{\prime}}, \eta_{\nu}^{\prime}\right)$ and $\left(N_{\nu}, J^{N_{\nu}}, \eta_{\boldsymbol{\nu}}\right)$ are isomorphic Kähler orbifolds. In particular, $\left(Y_{\boldsymbol{\nu}}, \beta_{\boldsymbol{\nu}}\right)$ is a contact orbifold.

Proof of Proposition 2.6. It suffices to prove that $\left(N_{\nu}^{\prime}, J^{N_{\nu}^{\prime}}, \eta_{\nu}^{\prime}\right)$ is a Kähler orbifold, since the other statements follow directly.

The uniformized tangent space of $Y_{\nu}$ splits as the direct sum $V\left(Y_{\nu}\right) \oplus$ $H\left(Y_{\nu}\right)$, where $V\left(Y_{\nu}\right)$ is the tangent space to the orbits of $\sigma^{Y_{\nu}}$. To check that $\eta_{\nu}$ is Kähler, it suffices therefore to verify that the restriction of $\mathrm{d} \beta_{\boldsymbol{\nu}}$ to $H\left(Y_{\boldsymbol{\nu}}\right)$ is compatible with the complex structure. In view of (44) and $T$-invariance, we need only check that the form

$$
Q_{\nu}^{*}\left(\mathrm{~d} \beta_{\boldsymbol{\nu}}\right)=\|\boldsymbol{\nu}\|^{2} \jmath_{\nu}^{*}\left(\frac{1}{\Phi^{\nu}} \mathrm{d} \alpha-\frac{1}{\left(\Phi^{\nu}\right)^{2}} \mathrm{~d} \Phi^{\nu} \wedge \alpha\right)
$$

where $Q_{\nu}$ is as in (37) and $\jmath_{\nu}$ as in (41), is compatible with the complex structure of the CR bundle $\mathcal{H}\left(X_{\nu}\right)$.

Suppose $x \in X_{\nu}$ and let $m:=\pi(x) \in M_{\nu}$. The general vector in $\mathcal{H}\left(X_{\nu}\right)_{x}$ has the form $v^{\sharp}$ for some $v \in \mathcal{H}\left(M_{\nu}\right)_{m}$ (see (25) ), and then $J_{x}^{\prime}\left(v^{\sharp}\right)=J_{m}(v)^{\sharp}$. By (45), for any $v, w \in \mathcal{H}\left(M_{\nu}\right)_{m}$

$$
Q_{\nu}^{*}\left(\mathrm{~d} \beta_{\boldsymbol{\nu}}\right)_{x}\left(v^{\sharp}, w^{\sharp}\right)=\frac{\|\boldsymbol{\nu}\|^{2}}{\Phi^{\nu}(m)} \mathrm{d}_{x} \alpha\left(v^{\sharp}, w^{\sharp}\right)=\frac{\|\boldsymbol{\nu}\|^{2}}{\Phi^{\nu}(m)} 2 \omega_{m}(v, w) .
$$

The statement follows, since $\Phi^{\nu}(m)>0$ by definition of $M_{\nu}, \mathcal{H}_{m}\left(M_{\boldsymbol{\nu}}\right) \subseteq$ $T_{m} M$ is a complex subspace, and $\omega$ is Kähler.

Corollary 2.19. $\left(N_{\boldsymbol{\nu}}, B_{\boldsymbol{\nu}}\right)$ is polarized Kähler orbifold.

Here notation is as in Chaper 4 of [BG]. By the Kodaira-Baily Vanishing Theorem ([B], $[\mathrm{BG}])$, we obtain the following conclusion.

Corollary 2.20. $H^{i}\left(N_{\nu}, B_{k \nu}\right)=0, \forall i>0, k \gg 0$.

\subsection{An Hamiltonian circle action on $N_{\nu}$}

The action $\rho^{X}: S^{1} \times X \rightarrow X$ with infinitesimal generator $-\partial_{\theta}$ in (1) is the contact lift of the trivial circle action on $M$ corresponding to the moment map $\Phi=1$ (recall (4)). We shall see that $\rho^{X}$ determines a contact action on $\left(Y_{\boldsymbol{\nu}}, \beta_{\boldsymbol{\nu}}\right)$ and an holomorphic Hamiltonian action on $\left(N_{\boldsymbol{\nu}}^{\prime}, J^{N_{\boldsymbol{\nu}}^{\prime}}, 2 \eta_{\boldsymbol{\nu}}^{\prime}\right)$, such 
that the former is the contact lift of the latter by (the orbifold version of) the procedure in (4), when we regard $Y_{\nu}$ as an orbifold circle bundle on $X_{\nu}$.

Clearly, $\rho^{X}$ commutes with $\mu^{X}$. Hence $\rho^{X}$ leaves $X_{\nu}$ invariant and determines a restricted action $\rho^{X_{\nu}}: S^{1} \times X_{\nu} \rightarrow X_{\nu}$. For the same reason $\rho^{X_{\nu}}$ passes to the quotients $Y_{\nu}$ and $N_{\nu}$. In other words, $\rho^{X_{\nu}}$ descends to actions $\rho^{Y_{\nu}}: S^{1} \times Y_{\nu} \rightarrow Y_{\nu}$ and $\rho^{N_{\nu}^{\prime}}: S^{1} \times N_{\nu}^{\prime} \rightarrow N_{\nu}^{\prime}$, so that the projections $Q_{\nu}: X_{\nu} \rightarrow Y_{\nu}$ and $\pi_{\nu}: Y_{\nu} \rightarrow N_{\nu}^{\prime}$ are equivariant.

In particular, if $-\partial_{\theta}^{X_{\nu}}$ is the restriction of $-\partial_{\theta}$ to $X_{\nu},-\partial_{\theta}^{Y_{\nu}}$ is the infinitesimal generator of $\rho^{Y_{\nu}}$, and $-\partial_{\theta}^{N_{\nu}}$ is the infinitesimal generator of $\rho^{N_{\nu}}$, then $\partial_{\theta}^{X_{\nu}}$ and $\partial_{\theta}^{Y_{\nu}}$ are $Q_{\nu}$-related, and similarly $\partial_{\theta}^{Y_{\nu}}$ and $\partial_{\theta}^{N_{\nu}}$ are $\pi_{\nu}$-related.

Lemma 2.11. $\rho^{N_{\nu}^{\prime}}$ is Hamiltonian on $\left(N_{\boldsymbol{\nu}}^{\prime}, 2 \eta_{\boldsymbol{\nu}}^{\prime}\right)$, with moment map $\|\boldsymbol{\nu}\|^{2} / \bar{\Phi}^{\nu}+$ $c$, for any $c \in \mathbb{R}$.

Proof of Lemma 2.11. By T-invariance of all terms involved, and the previous remark about the correlations of the generating vector fields, we need only prove that

$$
-\iota\left(\partial_{\theta}^{X}\right) Q_{\nu}^{*}\left(\mathrm{~d} \beta_{\nu}\right)=\mathrm{d}\left(\|\boldsymbol{\nu}\|^{2} / \Phi^{\nu} \circ \jmath_{\nu}\right),
$$

where $\jmath_{\boldsymbol{\nu}}$ is as in (41) and $Q_{\nu}^{*}\left(\mathrm{~d} \beta_{\boldsymbol{\nu}}\right)$ as in (45). We have on a neighborhood of $X_{\nu}$ :

$$
-\iota\left(\partial_{\theta}\right)\|\boldsymbol{\nu}\|^{2}\left(\frac{1}{\Phi^{\nu}} \mathrm{d} \alpha-\frac{1}{\left(\Phi^{\nu}\right)^{2}} \mathrm{~d} \Phi^{\nu} \wedge \alpha\right)=-\frac{\|\boldsymbol{\nu}\|^{2}}{\left(\Phi^{\nu}\right)^{2}} \mathrm{~d} \Phi^{\nu}=\mathrm{d}\left(\frac{\|\boldsymbol{\nu}\|^{2}}{\Phi^{\nu}}\right),
$$

establishing the claim.

Thus $-\partial_{\theta}^{N_{\nu}^{\prime}}$ is a Hamiltonian vector field on $\left(N_{\nu}^{\prime}, 2 \eta_{\nu}^{\prime}\right)$, and every choice of $c \in \mathbb{R}$ in Lemma 2.11 determines a contact lift $-\widehat{\partial_{\theta}^{N_{\nu}^{\prime}}}$ (implicitly depending on $c)$ to $\left(Y_{\boldsymbol{\nu}}, \beta_{\boldsymbol{\nu}}\right)$ of $-\partial_{\theta}^{N_{\nu}^{\prime}}$, as in (41).

Here $Y_{\nu}$ plays the role of $X, \beta_{\nu}$ the role of $\alpha$, and $-\partial_{\theta}^{N_{\nu}^{\prime}}$ the one of $\xi_{M}$. The role of $-\partial_{\theta}$ (the infinitesimal generator of $\rho^{X}$ ) is played by $-\delta^{Y_{\nu}}$ (the infinitesimal generator of $\left.\sigma^{Y_{\nu}}\right)$.

We need to determine the 'correct choice' of $c$ that determines $\rho^{Y_{\nu}}$ as the contact lift of $\rho^{N_{\nu}^{\prime}}$.

Lemma 2.12. We have $\widetilde{\partial_{\theta}^{N_{\nu}^{\prime}}}=\partial_{\theta}^{Y_{\nu}}$ if and only if $c=0$.

Given an (orbifold) vector field $V$ on $N_{\boldsymbol{\nu}}$, we shall denote by $V^{\natural} \in \mathfrak{X}\left(Y_{\boldsymbol{\nu}}\right)$ its horizontal lift to $Y_{\boldsymbol{\nu}}$ with respect to $\beta_{\boldsymbol{\nu}}$. 
Proof of Lemma 2.12. On $X_{\boldsymbol{\nu}}$ we have by (4)

$$
\frac{1}{\|\boldsymbol{\nu}\|^{2}} \boldsymbol{\nu}_{X_{\nu}}=\frac{1}{\|\boldsymbol{\nu}\|^{2}} \boldsymbol{\nu}_{M_{\nu}}^{\sharp}-\frac{\Phi^{\nu}}{\|\boldsymbol{\nu}\|^{2}} \partial_{\theta}^{X_{\nu}} .
$$

Here $\boldsymbol{\nu}_{M_{\nu}}$ is the restriction of $\boldsymbol{\nu}_{M}$ to $M_{\boldsymbol{\nu}}$ (a vector field on $M_{\boldsymbol{\nu}}$ ), and $\boldsymbol{\nu}_{M_{\boldsymbol{\nu}}}^{\sharp}$ is its horizontal lift to $X_{\nu}$.

Given that $\rho^{X}$ and $\mu^{X}$ commute, $\left[\boldsymbol{\nu}_{X}, \partial_{\theta}\right]=0$ on $X$; this implies $\left[\boldsymbol{\nu}_{M}^{\sharp}, \boldsymbol{\nu}_{X}\right]=$ $\left[\boldsymbol{\nu}_{M}^{\sharp}, \partial_{\theta}\right]=0$. Furthermore, one has $\left[\boldsymbol{\nu}_{X}, \boldsymbol{\gamma}_{X}\right]=0$ for every $\boldsymbol{\gamma} \in \mathfrak{t}$, and this implies also $\left[\boldsymbol{\nu}_{M}^{\sharp}, \boldsymbol{\gamma}_{X}\right]=0$.

Being horizontal and $T_{\nu^{\perp}}^{r-1}$-invariant, $\boldsymbol{\nu}_{M_{\nu}}^{\sharp} / \Phi^{\nu}$ is $\pi_{\nu^{\prime}}$-related to a horizontal vector field on $Y_{\nu}$; the latter is $\sigma^{Y^{\nu}}$-invariant by the above, and therefore it is the horizontal lift $-v^{\natural}$ to $Y_{\nu}$ of a vector field $-v$ on $N_{\nu}$.

Multiplying both sides of (47) by $\|\boldsymbol{\nu}\|^{2} / \Phi^{\nu}$ and then pushing down to $Y_{\boldsymbol{\nu}}$ we obtain

$$
v^{\natural}-\frac{\|\boldsymbol{\nu}\|^{2}}{\overline{\Phi^{\nu}}} \delta^{Y_{\nu}}=-\frac{1}{\overline{\Phi^{\nu}}} \boldsymbol{\nu}_{M_{\nu}}^{\sharp}-\frac{\|\boldsymbol{\nu}\|^{2}}{\overline{\Phi^{\nu}}} \delta^{Y_{\nu}}=-\partial_{\theta}^{Y_{\boldsymbol{\nu}}},
$$

and pushing down to $N_{\nu}$ this yields

$$
v=-\partial_{\theta}^{N_{\nu}}
$$

In view of Lemma 2.11, (49) implies that $v$ is the Hamiltonian vector field on $\left(N_{\boldsymbol{\nu}}^{\prime}, 2 \eta_{\boldsymbol{\nu}}^{\prime}\right)$ of $\|\boldsymbol{\nu}\|^{2} / \bar{\Phi}^{\boldsymbol{\nu}}+c$; then (48) implies that $-\partial_{\theta}^{Y_{\nu}}$ is its contact lift corresponding to $c=0$. It is clear that any other choice of $c$ yields a different lift.

In the following, we shall identify the pairs $\left(N_{\nu}^{\prime}, B_{k \nu}^{\prime}\right) \cong\left(N_{\nu}, B_{k \nu}\right)$.

\subsection{The Fourier decomposition of $\mathcal{O}\left(\tilde{A}_{\nu}^{\vee}\right)_{k \nu}$}

Consider the holomorphic action

$$
\rho^{A_{0}^{\vee}}:\left(e^{\imath \theta}, \ell\right) \in S^{1} \times A_{0}^{\vee} \rightarrow e^{-\imath \theta} \ell \in A_{0}^{\vee} .
$$

Thus $\rho^{A_{0}^{\vee}}$ extends $\rho^{X}$. Similarly, let $\mu^{A_{0}^{\vee}}: T \times A_{0}^{\vee} \rightarrow A_{0}^{\vee}$ be the holomorphic action extending $\mu^{X}$. Clearly, $\rho^{A_{0}^{\vee}}$ and $\mu^{A_{0}^{\vee}}$ commute.

The dense open subset $\tilde{A}_{\nu}^{\vee} \subseteq A_{0}^{\vee}$ is invariant under both $\rho^{A_{0}^{\vee}}$ and $\mu^{A_{0}^{\vee}}$, which therefore restrict to commuting holomorphic actions $\rho^{\tilde{A}_{\nu}^{\vee}}$ and $\mu^{\tilde{A}_{\nu}^{\vee}}$ on $\tilde{A}_{\nu}^{\vee}$. 
Therefore, $\rho^{\tilde{A}_{\nu}^{\vee}}$ and $\mu^{\tilde{A}_{\nu}^{\vee}}$ determine commuting representations $\hat{\rho}^{\tilde{A}_{\nu}^{\vee}}$ of $S^{1}$ and $\hat{\mu}^{\vee} \tilde{A}_{\nu}^{\vee}$ of $T$ on the space $\mathcal{O}\left(\tilde{A}_{\nu}^{\vee}\right)$ of holomorphic functions on $\tilde{A}_{\nu}^{\vee}$, given by

$$
\hat{\rho}_{e^{\imath \theta}}^{\tilde{A}_{\vee}^{\vee}}(s):=s \circ \rho_{e^{-\imath \theta}}^{\tilde{A}_{\nu}}, \quad \hat{\mu}_{\mathbf{t}}^{\tilde{A}_{\nu}^{\vee}}(s):=s \circ \mu_{\mathbf{t}^{-1}}^{\tilde{A}_{\vee}^{\vee}} \quad\left(s \in \mathcal{O}\left(\tilde{A}_{\nu}^{\vee}\right), e^{\imath \theta} \in S^{1}, \mathbf{t} \in T .\right) .
$$

For every $l \in \mathbb{Z}$ and $\boldsymbol{\lambda} \in \mathbb{Z}^{r}$, let $\mathcal{O}\left(\tilde{A}_{\nu}^{\vee}\right)_{l}$ and $\mathcal{O}\left(\tilde{A}_{\nu}^{\vee}\right)_{\boldsymbol{\lambda}}$ be the $l$-th and $\boldsymbol{\lambda}$-th isotypical components of $\mathcal{O}\left(\tilde{A}_{\nu}^{\vee}\right)$, respectively, for $\hat{\rho}^{\tilde{A}_{\nu}^{\vee}}$ and $\hat{\mu}^{\tilde{A}_{\nu}^{\vee}}$, respectively. Hence $\hat{\rho}^{\tilde{A}_{\nu}^{\vee}}$ restricts to a subrepresentation on $\mathcal{O}\left(\tilde{A}_{\nu}^{\vee}\right)_{\lambda}$. In particular, for every $k=1,2, \ldots$ the vector space $\mathcal{O}\left(\tilde{A}_{\nu}^{\vee}\right)_{k \nu}$ is finite dimensional by (40), and we have an $S^{1} \times T$-equivariant decomposition

$$
\mathcal{O}\left(\tilde{A}_{\nu}^{\vee}\right)_{k \nu}=\bigoplus_{l \in \mathbb{Z}} \mathcal{O}\left(\tilde{A}_{\nu}^{\vee}\right)_{k \nu, l}
$$

where $\mathcal{O}\left(\tilde{A}_{\boldsymbol{\nu}}^{\vee}\right)_{k \boldsymbol{\nu}, l}=\mathcal{O}\left(\tilde{A}_{\boldsymbol{\nu}}^{\vee}\right)_{k \boldsymbol{\nu}} \cap \mathcal{O}\left(\tilde{A}_{\boldsymbol{\nu}}^{\vee}\right)_{l}$. Since the isomorphisms in (40) are by construction $S^{1}$-equivariant, (50) may be interpreted in terms of $H^{0}\left(N_{\nu}, B_{k \nu}\right)$ :

$$
H^{0}\left(N_{\boldsymbol{\nu}}, B_{k \boldsymbol{\nu}}\right)=\bigoplus_{l \in \mathbb{Z}} H^{0}\left(N_{\boldsymbol{\nu}}, B_{k \boldsymbol{\nu}}\right)_{l} .
$$

Lemma 2.13. If $k \gg 0, H^{0}\left(N_{\nu}, B_{k \nu}\right)_{l}=0$ for all $l \leq 0$.

Proof of Lemma [2.13. In the terminology of [MS], the datum of the Hamiltonian action $\rho^{N_{\nu}}$, with moment map $\|\boldsymbol{\nu}\|^{2} / \overline{\Phi^{\nu}}$, makes $B_{k \nu}$ into a prequantum $S^{1}$-equivariant orbibundle, hence into a moment line bundle. By Corollary 2.11 of [MS], and given that $\|\boldsymbol{\nu}\|^{2} / \overline{\Phi^{\nu}}>0$, we conclude that the Fourier decomposition of $\operatorname{RR}\left(N_{\nu}, B_{k \nu}\right)$ (viewed as a virtual character of $S^{1}$ ) has the form

$$
\operatorname{RR}\left(N_{\boldsymbol{\nu}}, B_{k \boldsymbol{\nu}}\right)=\sum_{l>0} \operatorname{RR}\left(N_{\boldsymbol{\nu}}, B_{k \boldsymbol{\nu}}\right)_{l} \cdot \chi_{l},
$$

where where $\chi_{l}\left(e^{\imath \theta}\right)=e^{\imath l \theta}$. In view of Corollary 2.20 this means that, as a representation of $S^{1}$,

$$
H^{0}\left(N_{\boldsymbol{\nu}}, B_{k \boldsymbol{\nu}}\right)=\bigoplus_{l>0} H^{0}\left(N_{\boldsymbol{\nu}}, B_{k \boldsymbol{\nu}}\right)_{l} \quad \forall k \gg 0 .
$$

By the $S^{1}$-equivariance in (40), we can now sharpen (50) as follows.

Corollary 2.21. If $k \gg 0$, then

$$
\mathcal{O}\left(\tilde{A}_{\nu}^{\vee}\right)_{k \nu}=\bigoplus_{l>0} \mathcal{O}\left(\tilde{A}_{\nu}^{\vee}\right)_{k \nu, l}
$$




\section{Proof of Theorem 1.1}

We can now give the proof of Theorem 1.1. First, however, let us consider the following statement.

Lemma 3.1. For every $\boldsymbol{\lambda} \in \mathbb{Z}$, restriction yields an isomorphism $\mathcal{O}\left(A_{0}^{\vee}\right)_{\boldsymbol{\lambda}} \cong$ $H(X)_{\lambda}^{\hat{\mu}}$.

Proof of Lemma 3.1. Clearly, restriction yields a morphism $\zeta_{\boldsymbol{\lambda}}: \mathcal{O}\left(A_{0}^{\vee}\right)_{\boldsymbol{\lambda}} \rightarrow$ $H(X)_{\lambda}^{\hat{\mu}}$. If $f \in \mathcal{O}\left(A_{0}^{\vee}\right)$ is non-zero, then the locus where its differential vanishes has real codimension $\geq 2$; if it vanishes on $X$, therefore, $f=0$. Hence $\zeta_{\boldsymbol{\lambda}}$ is injective.

Since by assumption $\mathbf{0} \notin \Phi(M)$, we have $\operatorname{dim} H(X)_{\boldsymbol{\lambda}}^{\hat{\mu}}<+\infty$ for every $\boldsymbol{\lambda}$. Hence we have a finite direct sum

$$
H(X)_{\boldsymbol{\lambda}}^{\hat{\mu}}=\bigoplus_{l=a(\boldsymbol{\lambda})}^{b(\boldsymbol{\lambda})} H(X)_{\boldsymbol{\lambda}, l}^{\hat{\mu}},
$$

where

$$
0 \leq a(\boldsymbol{\lambda}) \leq b(\boldsymbol{\lambda})<+\infty, \quad H(X)_{\boldsymbol{\lambda}, l}^{\hat{\mu}}:=H(X)_{\boldsymbol{\lambda}}^{\hat{\mu}} \cap H(X)_{l} .
$$

Hence, to verify that $\zeta_{\boldsymbol{\lambda}}$ is surjective, it suffices to show that any $s \in H(X)_{\boldsymbol{\lambda}, l}^{\hat{\mu}}$ is the restriction of some $\tilde{s} \in \mathcal{O}\left(A_{0}^{\vee}\right)_{\lambda}$. Any $s \in H(X)_{l}^{\hat{\mu}}$ is the restriction of an holomorphic homogeneous function of degree $l, \tilde{s} \in \mathcal{O}\left(A_{0}^{\vee}\right)_{l}$. Since $\rho^{A_{0}^{\vee}}$ and $\gamma^{A_{0}^{\vee}}$ commute, one sees that $\tilde{s}$ is in the $\boldsymbol{\lambda}$-th isotype for $T$, and therefore for $\tilde{T}$ as well. Hence $\zeta_{\boldsymbol{\lambda}}$ is surjective.

Proof of Theorem 1.1. By Lemma 3.1, for every $k=1,2, \ldots$ we have a natural equivariant injective linear map

$$
F_{k \nu}:=\operatorname{res}_{k \boldsymbol{\nu}} \circ \zeta_{k \nu}^{-1}: H(X)_{k \boldsymbol{\nu}}^{\hat{\mu}} \rightarrow \mathcal{O}\left(\tilde{A}_{\boldsymbol{\nu}}^{\vee}\right)_{k \boldsymbol{\nu}} \cong H^{0}\left(N_{\boldsymbol{\nu}}, B_{k \boldsymbol{\nu}}\right)
$$

where $\operatorname{res}_{k \nu}: \mathcal{O}\left(A_{0}^{\vee}\right)_{k \nu} \rightarrow \mathcal{O}\left(\tilde{A}_{\nu}^{\vee}\right)_{k \nu}$ denotes restriction, and is obviously injective since $\tilde{A}_{\nu}^{\vee}$ is open and dense in $A_{0}^{\vee}$; this proves the first statement of Theorem 1.1.

To prove the second statement, it suffices to verify that $r e s_{k \nu}$ is surjective for $k \gg 0$. We have for some $c(k, \boldsymbol{\nu}), d(k, \boldsymbol{\nu}) \in \mathbb{Z}$ with $c(k, \boldsymbol{\nu}) \leq d(k, \boldsymbol{\nu})$ :

$$
\mathcal{O}\left(\tilde{A}_{\boldsymbol{\nu}}^{\vee}\right)_{k \boldsymbol{\nu}}=\bigoplus_{l=c(k, \boldsymbol{\nu})}^{d(k, \boldsymbol{\nu})} \mathcal{O}\left(\tilde{A}_{\boldsymbol{\nu}}^{\vee}\right)_{k \boldsymbol{\nu}, l}
$$


hence

$$
r e s_{k \boldsymbol{\nu}}=\bigoplus_{l=c(k, \boldsymbol{\nu})}^{d(k, \boldsymbol{\nu})} r e s_{k \boldsymbol{\nu}, l},
$$

where

$$
\operatorname{res}_{k \boldsymbol{\nu}, l}: \mathcal{O}\left(A_{0}^{\vee}\right)_{k \boldsymbol{\nu}, l} \rightarrow \mathcal{O}\left(\tilde{A}_{\boldsymbol{\nu}}^{\vee}\right)_{k \boldsymbol{\nu}, l}
$$

and we need to check that $r e s_{k, l}$ is surjective for every $l=c(k, \boldsymbol{\nu}), \ldots, d(k, \boldsymbol{\nu})$ and $k \gg 0$.

By Corollary 2.21, we may assume that $c(k, \boldsymbol{\nu})>0$. Furthermore, by Lemma $2.4 \tilde{A}_{\nu}^{\vee}=\left(\pi^{\prime}\right)^{-1}\left(\tilde{M}_{\nu}\right)$ and therefore $\operatorname{res}_{k \boldsymbol{\nu}, l}$ may canonically reinterpreted in terms of the restriction of holomorphic sections:

$$
\widetilde{r e s}_{k \nu, l}: H^{0}\left(M, A^{\otimes l}\right)_{k \boldsymbol{\nu}} \rightarrow H^{0}\left(\tilde{M}_{\boldsymbol{\nu}}, A^{\otimes l}\right)_{k \boldsymbol{\nu}} .
$$

Hence we are reduced to proving that $\widetilde{r e s}_{k \nu, l}$ in (56) is surjective for all $l>0$.

Suppose $s \in H^{0}\left(M, A^{\otimes l}\right)$. Then $s \in H^{0}\left(M, A^{\otimes l}\right)_{k \nu}$ if and only if the following two conditions hold:

1. $s$ is $\gamma^{X}$-invariant, i.e., $s \in H^{0}\left(M, A^{\otimes l}\right)^{T^{\perp}{ }^{\perp-1}}$;

2. for any $e^{\imath \vartheta} \in S^{1}$,

$$
\hat{\mu}_{e^{\imath \vartheta \nu}}(s)=e^{\imath k\|\boldsymbol{\nu}\|^{2} \vartheta} s .
$$

In other words, we can identify $H^{0}\left(M, A^{\otimes l}\right)_{k \nu}$ with the $k\|\boldsymbol{\nu}\|^{2}$-isotypical component for the representation of $T_{\nu}^{1} \cong S^{1}$ on $H^{0}\left(M, A^{\otimes l}\right)^{T^{r-1}}$. The same considerations apply to $H^{0}\left(\tilde{M}_{\boldsymbol{\nu}}, A^{\otimes l}\right)_{k \boldsymbol{\nu}}$. We shall express this by writing

$$
H^{0}\left(M, A^{\otimes l}\right)_{k \nu}=H^{0}\left(M, A^{\otimes l}\right)_{k\|\nu\|^{2}}^{T_{\nu}^{r-1}}, \quad H^{0}\left(\tilde{M}_{\boldsymbol{\nu}}, A^{\otimes l}\right)_{k \boldsymbol{\nu}}=H^{0}\left(\tilde{M}_{\boldsymbol{\nu}}, A^{\otimes l}\right)_{k\|\boldsymbol{\nu}\|^{2}}^{T_{\nu}^{r-1}}
$$

It is well-known that the restriction map

$$
f_{l, \nu}: H^{0}\left(M, A^{\otimes l}\right)^{T_{\nu \perp}^{r-1}} \rightarrow H^{0}\left(\tilde{M}_{\nu}, A^{\otimes l}\right)^{T_{\nu}^{r-1}}
$$

is an isomorphism ( $\S 5$ of [GS], Theorem 2,18 of $[\mathrm{Sj}]$ ), and it is clearly $T_{\nu^{-}}^{1}$ equivariant. The claim follows, since $\widetilde{r e s}_{k \nu, l}$ is the restriction of $f_{l, \boldsymbol{\nu}}$ to the $k\|\boldsymbol{\nu}\|^{2}$-isotypical component, hence by equivariance it induces an isomor-

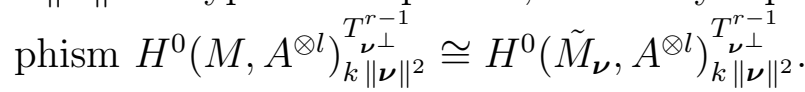




\section{References}

[ALR] A. Adem, J. Leida, Y. Ruan, Orbifolds and stringy topology, Cambridge Tracts in Mathematics, 171, Cambridge University Press, Cambridge, 2007

[B] W. L. Baily, On the imbedding of $V$-manifolds in projective space, Amer. J. Math. 79 (1957), 403-430

[BG] C. P. Boyer, K. Galicki, Sasakian geometry, Oxford Mathematical Monographs. Oxford University Press, Oxford, 2008

[Ca] S. Camosso, Scaling asymptotics of Szegö kernels under commuting Hamiltonian actions, Ann. Mat. Pura Appl. (4) 195 (2016), no. 6, 2027-2059

[Gr] H. Grauert, Über Modifikationen und exzeptionelle analytische Mengen, Math. Ann. 146 (1962), 331-368

[GS] V. Guillemin, S. Sternberg Geometric quantization and multiplicities of group representations, Invent. Math. 67 (1982), no. 3, 515538

[Ka] T. Kawasaki, The Riemann-Roch theorem for complex V-manifolds, Osaka Math. J. 16 (1979), no. 1, 151-159

[Ko] B. Kostant, Quantization and unitary representations. I. Prequantization, Lectures in modern analysis and applications, III, pp. 87208. Lecture Notes in Math., Vol. 170, Springer, Berlin, 1970

[LT] E. Lerman, S. Tolman Hamiltonian torus actions on symplectic orbifolds and toric varieties, Trans. Amer. Math. Soc. 349 (1997), no. 10,42014230

[MS] E. Meinrenken, R. Sjamaar, Singular reduction and quantization, Topology 38 (1999), no. 4, 699-762

[P1] R. Paoletti, Asymptotics of Szegö kernels under Hamiltonian torus actions, Israel Journal of Mathematics 191 (2012), no. 1, 363-403 DOI: $10.1007 / \mathrm{s} 11856-011-0212-4$

[P2] R. Paoletti, Lower-order asymptotics for Szegö and Toeplitz kernels under Hamiltonian circle actions, Recent advances in algebraic geometry, 321-369, London Math. Soc. Lecture Note Ser., 417, Cambridge Univ. Press, Cambridge, 2015 
[P3] R. Paoletti, Conic reductions for Hamitonian actions of $U(2)$ and its maximal torus, arXiv:2002.08105

[S1] I. Satake, On a generalization of the notion of manifold, Proc. Nat. Acad. Sci. U.S.A. 42 (1956), 359363

[S2] I. Satake, The Gauss-Bonnet theorem for V-manifolds, J. Math. Soc. Japan 9 (1957), 464492

[Sj] R. Sjamaar, Holomorphic slices, symplectic reduction and multiplicities of representations, Ann. of Math. (2) 141 (1995), no. 1, $87-129$

[St] S. Sternberg, Group theory and physics, Cambridge University Press, Cambridge, 1994 